\title{
Detection and sizing of single droplets flowing in a lab-on-a-chip device by measuring impedance fluctuations
}

\author{
Nour Eddin Yakdi, François Huet, Kieu Ngo ${ }^{*}$ \\ Sorbonne Universités, UPMC Univ Paris 06, CNRS, Laboratoire Interfaces et Systèmes \\ Electrochimiques (LISE), F-75005, Paris, France
}

\begin{abstract}
This paper is devoted to the evaluation of the electrochemical noise technique consisting in measuring the fluctuations of the electrolyte resistance (ER) between two metallic electrodes immerged in a conductive electrolyte to detect and characterize single particles circulating in a microfluidic device, without the help of optical measurements that require good visibility of the detection region. Numerical simulations were performed with the finite element method to study the influence of the dimensions of the channel and the electrodes on the ER. Measurements of the ER variations due to the passage of oil droplets and plugs passing between the electrodes were carried out. Excellent agreement was obtained between the theoretical and experimental ER transients, which allowed the velocity and diameter of the oil droplets to be estimated with an accuracy of a few percents in the case of droplet diameters ranging from 60 to $100 \mu \mathrm{m}$. According to the numerical simulations and the amplitude of the background noise, oil droplets of diameter larger than 20-25 $\mu \mathrm{m}$ can be detected in the microchannel used (cross section of $100 \mu \mathrm{m} \times 100 \mu \mathrm{m}$ and $100 \mu \mathrm{m} \times 100 \mu \mathrm{m}$ electrodes separated by a gap of $100 \mu \mathrm{m})$. Developments of smaller microfluidic devices are under progress to detect and characterize particles of a few micrometers, such as biological cells for example.
\end{abstract}

Keywords: Particle sizing techniques; Two-phase systems; Electrochemical noise; Impedance, Electrolyte resistance; microfluidic device; oil droplet

${ }^{*}$ Corresponding author. Tel.: +33-1-44279617; fax: 33-1-44274074

E-mail address: kieu.ngo@upmc.fr (K. Ngo) 


\section{Introduction}

Microfluidic systems offer some attractive advantages over standard macroscale techniques for advanced chemistry, material science, biology and medicine since they allow small sample volumes, high sensitivity, reduced analysis time and low fabrication costs [1-5]. These systems, with dimensions typically of tens to hundreds of micrometers, deal with continuous streams of fluids. In contrast, droplet-based microfluidics focuses on creating discrete droplets by mixing two immiscible fluids in a microfluidic channel, at a T-junction or using a flow-focusing technique, in order to enable rapid mixing, no reagent dispersion and high-throughput analysis [6-13]. Indeed, droplets with volumes ranging from femtoliters to nanoliters can be generated at high rates, up to 10,000 droplets per second [14], each droplet serving as an individual microreactor. Applications of droplet-based microfluidics have been demonstrated in the past decade for single-cell analysis [1517], drug delivery $[18,19]$, and diagnostics $[20,21]$.

Despite many efforts to develop specific devices for droplet generation and manipulation, including droplet moving, merging, mixing, sorting and trapping, the detection and characterization of single droplets remains a challenging task. To date, methods based on optical technologies are the most valuable techniques because of their numerous advantages, such as high sensitivity and fast response [2,14,22-26]. However, optical techniques are often bulky and expensive, and they require a good visibility of the detection volume so that they cannot be used in opaque media. Moreover, the droplets often need to be tagged with fluorescent markers. For these reasons, various non-optical methods have been developed [27,28], among which those using integrated microelectrodes for chronoamperometry [29-32], capacitive [33-35], or resistance measurements [36-42], are particularly interesting since they can be easily integrated with microchips. These techniques have been used to detect biological cells $[33,37,38,41,42]$, water-in-oil or oil-in-water droplets $[31,35,39]$, or to measure the concentration of various species within droplets $[30,32,40]$. Chronoamperometry consists in measuring the transient increases of the mass-transport limited current due to the passage of droplets in the diffusion layer of the working electrode, which requires 
the use of a reference electrode and the addition of an electro-active species in the continuous phase or in the droplets. In contrast, impedance spectroscopy is a label-free technique that does not require any reference electrode or electro-active species. Sun and Morgan reviewed in 2010 the different approaches and technologies employed for detecting and characterizing single cells with the impedance technique [36]. The impedance is usually measured at a single frequency using two facing or coplanar electrodes [38-40], or three coplanar electrodes $[37,41,42]$, or two pairs of facing electrodes, two of them on the same side of the microchannel being electrically coupled $[43,44]$. In all cases, the time records of the measured impedance parameter show transients indicating the passage of single cells. However, to the best of our knowledge, non-optical methods capable of precisely measuring a wide range of droplet size in a microchannel have not yet been developed.

Similar developments have been performed in electrochemistry with the electrochemical noise (EN) in two-phase flows consisting in measuring the fluctuations of current, potential or electrolyte resistance (ER) generated by the presence of a solid, liquid or gaseous phase dispersed in a conductive electrolyte. The EN technique is able to provide information on the elementary events at the origin of the fluctuations, such as the position, velocity, trajectory, and residence time of a particle in front of or in contact with a sensor $[45,46]$, the composition of an oil-in-water emulsion [47], the detachment frequency and size of evolving bubbles [48], the activity of pitting corrosion in multiphase flows [49], etc. For particles flowing in a channel, the ER fluctuations give direct information on the changes in electrolyte conductivity due to the passage of particles between the electrodes. The specific technique developed in our laboratory to measure the ER fluctuations between two electrodes [50] has been recently used to detect and characterize single particles of millimetric size flowing between two facing electrodes in a macrochannel [51]. This paper presents the necessary developments of the technique to measure the ER fluctuations and characterize droplets in a microfluidic channel. 


\section{Theoretical simulations}

The model of the microdevice is shown in Fig. 1. The electrolyte of conductivity $\kappa$ is contained in a cuboid of dimensions $l_{\mathrm{x}}, l_{\mathrm{y}}, l_{\mathrm{z}}$. Two identical metallic electrodes of dimensions $d_{\mathrm{x}}, l_{\mathrm{y}}$ are mounted flush in the floor of the channel at a distance $D$ between them. Compared to the size of the electrodes $\left(d_{\mathrm{x}}=l_{\mathrm{y}}=100 \mu \mathrm{m}\right)$ and the interelectrode distance $(D=100 \mu \mathrm{m})$ used in the experimental section, the $l_{\mathrm{x}}$ value was large enough $(1 \mathrm{~mm})$ to have no significant influence on the simulation results. The centre $\mathrm{O}$ of the Cartesian coordinates $x, y, z$ corresponds to the centre of the cuboid. The particle is an insulating sphere of diameter $d_{\mathrm{p}}$ that will flow along the $x$-axis in the experimental section. Its position is defined by the coordinates $x, y, z$ of its centre. The vertical position $z$ can also be defined as a function of the distance $h$ to the floor of the microchannel below the particle:

$$
z=h+d_{\mathrm{p}} / 2-l_{\mathrm{z}} / 2
$$

To calculate the ER between the two electrodes in the absence or in the presence of the particle, an electrical model was used, consisting in the application of a potential $\phi_{0}=30 \mathrm{mV}$ on one electrode while the other was grounded, the ER being calculated from the resulting current on the electrodes. The potential primary distribution, which does not depend on the (electro-)chemical reactions occurring on the electrode and on mass transport in the solution, was calculated at any point between the two electrodes by integrating Laplace's equation:

$$
\nabla^{2} \phi=0
$$

where $\phi$ is the electrical potential in the solution, with the following boundary condition at any point of the insulating surfaces (sphere and boundaries of the cuboid): the normal component of the current is equal to 0 so that the normal component of the potential gradient $\partial \phi / \partial \mathrm{n}$ is equal to 0 as well. This was done by using the finite element method with the COMSOL Multiphysics 4.1 software. To improve the accuracy of the simulation results, small mesh elements were used (maximum size of $1 \mu \mathrm{m}$ on the surfaces of the sphere and the electrodes instead of $55 \mu \mathrm{m}$ in the volume of the cuboid) and the maximum element growth rate was 1.3. 
The distribution of the current density, $J$, in the cuboid could then be determined according to the following expression:

$$
J=-\kappa \nabla \phi
$$

As an example, Fig. 2 presents the distribution of the current density in the presence of an insulating sphere. It may be noted that the current density is the highest in the inner parts of the electrodes $(|x|$ slightly higher than $D / 2$ ). The current lines are strongly deflected by the sphere so that high values of current density may be observed as well just above and below the sphere in the plane $y=0$.

The ER between the two electrodes can be determined from the ratio $\phi_{0} / I$, where the current I flowing between the electrodes is calculated by integrating the current density on the surface of the non-grounded electrode. Two parameters are now introduced to analyse the ER variations: $\Delta R_{\mathrm{e}}$ is the variation of the ER due to the presence of the sphere and $\Delta R_{\mathrm{e}, \text { norm }}$ is the normalized ER variation in percentage, defined as:

$$
\Delta R_{\mathrm{e}, \text { norm }}=\frac{\Delta R_{\mathrm{e}}}{R_{\mathrm{ea}}} \times 100
$$

where $R_{\mathrm{ea}}$ is the ER in the absence of the sphere between the electrodes.

For the microchannel of dimensions $l_{\mathrm{x}}, l_{\mathrm{y}}, l_{\mathrm{z}}$, and the configuration of the electrodes used in the experimental part (electrode dimensions $d_{\mathrm{x}}, l_{\mathrm{y}}$ and interelectrode distance $D$ ), $\Delta R_{\mathrm{e} \text {,norm }}$ depends on 4 parameters, the position $(x, y, z)$ of the sphere centre and the sphere diameter $d_{\mathrm{p}}$. To assess the possibility to determine the sphere diameter in single-particle sizing experiments, the influence of $x$, $y, z$, and $d_{\mathrm{p}}$ on the ER is presented below.

\subsection{Influence of the position of the insulating sphere}

The influence of the sphere position on the ER is shown in Fig. 3 for a sphere of diameter 50 $\mu \mathrm{m}$ (half of the vertical size) located at various positions $x$ and $z$ in the flow channel direction $(y=$ 0 ). It can be noticed that $R_{\mathrm{e}}$ starts increasing a little before the sphere enters the detection region $(-150 \mu \mathrm{m}<x<150 \mu \mathrm{m})$, indicating that the current lines are already modified by the presence of 
the sphere. The ER is also influenced by the vertical position of the sphere. The highest values are obtained when the sphere is located close to the floor of the microchannel because of the stronger constriction of the current lines between the sphere and the electrodes. When the distance $h$ below the sphere is $1 \mu \mathrm{m}$, the transient $\Delta R_{\mathrm{e}}(x)$ presents the shape of a camel's back with two humps positioned at the inner edges of the electrodes $(|x| \approx D / 2)$. This specific shape was already obtained in previous publications for small spheres flowing above disk electrodes $[46,52,53]$. The two humps are due to the screening effect of the sphere in regions where the current lines are more concentrated. When the distance below the sphere increases, the two distinct humps disappear progressively and the amplitude of the resulting hump decreases. In the experiments described below, no transient with a camel's back shape was observed and the maximum of the $\Delta R_{\mathrm{e}}(x)$ transients was always obtained at $x=0$, indicating that the spheres passed above the electrodes at a vertical position close to the middle of the channel $(z \approx 0)$. Hence, for simplification, $\Delta R_{\mathrm{e}}(x=0)$ is termed $\Delta R_{\mathrm{e}, \max }$ even if it is not the maximum amplitude in the case of the camel's back shape. As shown in Fig. 3, $\Delta R_{\mathrm{e}, \max }$ varies from $17.8 \Omega$ to $32.8 \Omega$ when the sphere of diameter $50 \mu \mathrm{m}$ is located close to the top of the channel $(h=49 \mu \mathrm{m})$ or to the floor $(h=1 \mu \mathrm{m})$, which corresponds to normalized ER values $\Delta R_{\mathrm{e}, \max }$, norm of $4.3 \%$ and $7.8 \%$, respectively $\left(R_{\mathrm{ea}}=418.7 \Omega\right)$.

For a sphere of bigger size $\left(d_{\mathrm{p}}=85 \mu \mathrm{m}\right)$ located on the axis $y=0$, the ER variations are given in Fig. 4 as a function of the vertical position. No camel's back shape can be observed, even for small distance below the sphere $(h=0.5 \mu \mathrm{m})$. Much higher values of $\Delta R_{\mathrm{e}, \max }$ are obtained, from 145.8 $\Omega$ when the sphere is close to the top of the channel $(h=14.5 \mu \mathrm{m})$ to $183.0 \Omega$ when it is close to the floor $(h=0.5 \mu \mathrm{m})$, which corresponds to $\Delta R_{\mathrm{e}, \max }$, norm values of $34.8 \%$ and $43.7 \%$, respectively.

Results in Figs. 3 and 4 are obtained for a sphere located on the $y$ axis. Fig. 5 shows the influence of the parameter $y$ for a sphere of diameter $50 \mu \mathrm{m}$ positioned at $x=z=0$. Small ER variations can be observed: the $\Delta R_{\mathrm{e}}$ values are ranging between $22.0 \Omega(y=0)$ to $23.3 \Omega(y= \pm 24$ 
$\mu \mathrm{m})$ and vary by $0.47 \%$ in the middle of the channel $(|\mathrm{y}|<10 \mu \mathrm{m})$. These small ER variations, compared to those due to the $x$ and $z$ positions of the sphere in Fig. 3, can be explained by the fact that the width $l_{\mathrm{y}}$ of the electrodes is exactly that of the channel. As a consequence, it will be considered in the experimental part that the measured ER values do not depend on the $y$ position of the sphere.

\subsection{Influence of the sphere diameter}

The influence of the sphere diameter on the ER variations is shown in Fig. 6 for a sphere located at the centre of the microchannel $(x=y=z=0)$. In a log-log scale, $\Delta R_{\mathrm{e}, \max }$ varies linearly with the sphere diameter according to the following expression obtained with a linear correlation coefficient of 0.99996:

$$
\Delta R_{\mathrm{e}, \max }=1.402 \times 10^{-4} d_{\mathrm{p}}{ }^{3.051} \quad\left(d_{\mathrm{p}} \text { in } \mu \mathrm{m}, \Delta R_{\mathrm{e}, \max } \text { in } \Omega\right)
$$

when the sphere diameter is smaller than half the height of the channel $\left(d_{\mathrm{p}}<l_{\mathrm{z}} / 2\right)$. While this exponent close to 3 was already obtained in previous simulation works investigating the influence on the ER of spherical insulating objects close to an electrode $[52,54,55]$ or between 2 disk electrodes [51], no analytical expression has been derived yet to express $\Delta R_{\mathrm{e}, \max }$ as a function of the sphere diameter in the case of non-uniform current lines between the electrodes. However, for uniform current lines, as in a Coulter counter, DeBlois and Bean derived an analytical expression of the resistance increment due to a small insulating sphere in suspension in a conductive electrolyte that is proportional to the volume fraction of the sphere, and, therefore, to the cube of the sphere diameter [56].

In order to derive the diameter of spheres circulating in the microchannel from the measured ER transients, another expression was determined by fitting the whole $\Delta R_{\mathrm{e}, \max }$ curve in Fig. 6:

$$
\Delta R_{\mathrm{e}, \max }=1.402 \times 10^{-4} d_{\mathrm{p}}^{3.051}\left(1+6.01 \times 10^{-12} d_{\mathrm{p}}^{5.648}\right) \quad\left(d_{\mathrm{p}} \text { in } \mu \mathrm{m}, \Delta R_{\mathrm{e}, \max } \text { in } \Omega\right)
$$

This expression is valid for $d_{\mathrm{p}} \leq 94 \mu \mathrm{m}$, as shown in Fig. 6 . 


\subsection{Method for particle sizing}

Fig. 7 shows the influence of the vertical position of an insulating sphere on the ER variation $\Delta R_{\mathrm{e}, \max }(x=y=0)$ for various sphere diameters. Limits in the calculation accuracy can be seen for the smallest spheres: decreasing the mesh size on and close to the sphere and electrode surfaces would yield more precise results but it would require a prohibited calculation time. It can be seen that the influence of the sphere diameter is much more important than that of the vertical position. This is especially true as the sphere circulates close to the middle of the channel, which is often the case in practice. This result can be exploited to determine the size of a single sphere from the maximum amplitude $\Delta R_{\mathrm{e}, \max }$ of the measured ER variation at $x=0$. Indeed, as shown in Fig. 8 in which the $\Delta R_{\mathrm{e}, \max }$ curves calculated at $\mathrm{z}=0, \mathrm{z}=-10 \mu \mathrm{m}$, and $\mathrm{z}=10 \mu \mathrm{m}$ are plotted, any $\Delta R_{\mathrm{e}, \max }$ value corresponds to $d_{\mathrm{p}}$ values within a narrow range. As an example, around a $d_{\mathrm{p}}$ value of $50 \mu \mathrm{m}$, the estimation error on the sphere diameter is $\pm 2 \mu \mathrm{m}$, which gives a relative error of $4 \%$. This error, which depends slightly on the sphere diameter $\left(4.6 \%\right.$ for $d_{\mathrm{p}}=15 \mu \mathrm{m}$ and $3.7 \%$ for $\left.d_{\mathrm{p}}=70 \mu \mathrm{m}\right)$, is proportional to the amplitude of the $z$ range $(2 \%$ for $[-5 \mu \mathrm{m}, 5 \mu \mathrm{m}]$ and $7.7 \%$ for $[-20 \mu \mathrm{m}, 20 \mu \mathrm{m}]$, both values given for $\left.d_{\mathrm{p}}=50 \mu \mathrm{m}\right)$.

As mentioned above, for the dimensions of the microchannel and the electrodes used in the experimental part, the ER variation $\Delta R_{\mathrm{e}}$ induced by the passage of a single spherical particle depends on 4 parameters, the position $(x, y, z)$ of the particle centre and the particle diameter $d_{\mathrm{p}}$. However, according to Fig. 5, when the particle flows close to the middle of the channel, the influence of the lateral position $y$ on $\Delta R_{\mathrm{e}}$ is not significant, so that $\Delta R_{\mathrm{e}}$ depends only on $x, z$ and $d_{\mathrm{p}}$. The following empirical expression of $\Delta R_{\mathrm{e}}$ was determined as a function of $x$ by fitting the $\Delta R_{\mathrm{e}}(x)$ curve calculated for $z=0$ and $d_{\mathrm{p}}=85 \mu \mathrm{m}$ (Fig. 4):

$$
\Delta R_{\mathrm{e}}\left(x, \mathrm{z}=0, d_{\mathrm{p}}=85 \mu \mathrm{m}\right)=\frac{\Delta R_{\mathrm{e}, \max }}{\left(1+4.574 \times 10^{-8}|x|^{3.892}\right)\left(1+5.491 \times 10^{-5}|x|^{1.937}\right)} \quad(x \text { in } \mu \mathrm{m})
$$


$\Delta R_{\mathrm{e}, \max }$ was then replaced by the expression given in Eq. 6 as a function of $d_{\mathrm{p}}$. As shown in Fig. 9, the following equation fits quite well the calculated $\Delta R_{\mathrm{e}}\left(x, z=0, d_{\mathrm{p}}\right)$ curve for various sphere diameters.

$$
\Delta R_{\mathrm{e}}\left(x, \mathrm{z}=0, d_{\mathrm{p}}\right)=\frac{1.402 \times 10^{-4} d_{\mathrm{p}}^{3.051}\left(1+6.01 \times 10^{-12} d_{\mathrm{p}}^{5.648}\right)}{\left(1+4.574 \times 10^{-8}|x|^{3.892}\right)\left(1+5.491 \times 10^{-5}|x|^{1.937}\right)} \quad\left(x \text { and } d_{\mathrm{p}} \text { in } \mu \mathrm{m}\right)
$$

To complete the theoretical simulations, Fig. 10 shows the influence of the sphere conductivity, $\kappa_{\mathrm{p}}$, on the electrolyte resistance variations. Two regions may be identified around point A where the sphere and electrolyte conductivities are equal $\left(\kappa_{\mathrm{p}}=\kappa=45.5 \mathrm{~S} / \mathrm{m}\right)$. For such value of $\kappa_{\mathrm{p}}$, the current lines are not modified by the presence of the sphere, which cannot be detected. When $\kappa_{\mathrm{p}}$ decreases below $\kappa, \Delta R_{\mathrm{e}}$ is positive and quickly attains its maximum value, $\Delta R_{\mathrm{e}, \max }$, obtained for insulating spheres $\left(\Delta R_{\mathrm{e}} / \Delta R_{\mathrm{e}, \max }=84.8 \%\right.$ and $98.4 \%$ for $\kappa=4.55$ and 0.455 $\mathrm{S} / \mathrm{m}$, respectively). In contrast, when $\kappa_{\mathrm{p}}$ increases above $\kappa, \Delta R_{\mathrm{e}}$ is negative and its absolute value rapidly reaches its maximum obtained for metallic spheres $\left(\Delta R_{\mathrm{e}} / \Delta R_{\mathrm{e}, \max }=77.4 \%\right.$ and $97.4 \%$ for $\kappa=$ 455 and $4550 \mathrm{~S} / \mathrm{m}$, respectively). In that case, the ER transients are in the negative direction. It should be noticed that $\Delta R_{\mathrm{e}, \max }$ in absolute value is higher for a conductive sphere than for an insulating one since the current lines can flow directly inside a conductive sphere while there are deviated around an insulating sphere. In practice, for detecting spherical particles of given conductivity $\kappa_{\mathrm{p}}$, it is sufficient to choose an electrolyte of conductivity slightly different from $\kappa_{\mathrm{p}}$. As shown in Fig. $10, \Delta R_{\mathrm{e}} / \Delta R_{\mathrm{e}, \max }$ is already equal to $38.2 \%$ when $\kappa=\kappa_{\mathrm{p}} / 2$ and $27.6 \%$ when $\kappa=2$ $\kappa_{\mathrm{p}}$.

\section{Experimental}

\subsection{Fabrication of the device}

The device used in this work (Fig. 11) consists of an inlet to the main channel (width $50 \mu \mathrm{m}$, height $100 \mu \mathrm{m}$ ) for the continuous phase (electrolyte), and several inlets to secondary channels of 
different width (5 to $50 \mu \mathrm{m}$ ) in which the dispersed phase (oil) was injected. Oil droplets generated at the junctions of the secondary and main channels flowed to the detection channel of larger width $(100 \mu \mathrm{m})$ and same height $(100 \mu \mathrm{m})$. The device configuration was chosen to generate droplets of various sizes in the detection channel, in which several pairs of gold electrodes of dimension $d_{\mathrm{x}}=$ $100 \mu \mathrm{m}$ and interelectrode distance $D=100 \mu \mathrm{m}$ were positioned to measure the ER fluctuations at various locations.

Microelectrodes were fabricated on a glass substrate using a standard lift-off process. A borosilicate glass substrate of diameter $10.24 \mathrm{~cm}$ and thickness $700 \mu \mathrm{m}$ was cleaned by immersion in a "piranha" solution $\left(\mathrm{H}_{2} \mathrm{SO}_{4} / \mathrm{H}_{2} \mathrm{O}_{2} 3: 1\right)$ for $30 \mathrm{~min}$ and dehydrated on a hot plate at $200^{\circ} \mathrm{C}$ for 30 min. Reversal photoresist AZ-5214 (Clariant) was spin-coated on the glass substrate at $800 \mathrm{rpm}$ for $10 \mathrm{~s}$ and 4,000 rpm for $30 \mathrm{~s}$. The photoresist layer was soft-baked at $110{ }^{\circ} \mathrm{C}$ for $1 \mathrm{~min}$ and exposed to ultraviolet (UV) light $\left(8 \mathrm{~mJ} / \mathrm{cm}^{2}\right)$ through a photomask using a EVG 620 mask aligner. A reversal bake was carried out at $120^{\circ} \mathrm{C}$ for 2 min before a flood exposure of UV without photomask (200 $\mathrm{mJ} / \mathrm{cm}^{2}$ ). In that way, the AZ-5214 resin was used as a negative photoresist to get the negative of the mask pattern. The photoresist patterns were then developed in 1:1 diluted AZ developer for $30 \mathrm{~s}$, briefly washed with deionised water, and dried under nitrogen. A gold layer of thickness $200 \mathrm{~nm}$ was deposited onto the glass substrate with a thin layer of $\mathrm{Cr}$ (10 nm thickness) used to promote adhesion between $\mathrm{Au}$ and glass. Both layers were deposited in a Plassys MEB 550S evaporator. Finally, the cross-linked photoresist was removed with acetone in an ultrasound bath.

The microfluidic channels were fabricated using standard soft lithography technique. The mold was built using SU-8 2100 (Microchem) and photopatterned on a silicon wafer using photolithography. The Si wafer was cleaned with acetone, isopropyl alcohol and "piranha". The silicon oxide layer was taken off in a hydrofluoric acid bath for $1 \mathrm{~min}$. The SU-8 resin was spincoated at $500 \mathrm{rpm}$ for $90 \mathrm{~s}$ and $3000 \mathrm{rpm}$ for $30 \mathrm{~s}$ to form a $100 \mu \mathrm{m}$ layer. A soft bake was performed at $65^{\circ} \mathrm{C}$ for $5 \mathrm{~min}$ and $95^{\circ} \mathrm{C}$ for $20 \mathrm{~min}$ before UV light exposition through a photomask $\left(250 \mathrm{~mJ} / \mathrm{cm}^{2}\right)$. A post-exposure bake was done at $65^{\circ} \mathrm{C}$ for $5 \mathrm{~min}$ and $95{ }^{\circ} \mathrm{C}$ for $10 \mathrm{~min}$ before the 
development in the SU-8 developer for $15 \mathrm{~min}$. A pattern height of $97.8 \mu \mathrm{m}$ was measured using a mechanic profilometer (Dektak). Polydimethylsiloxane (PDMS) mixed with the prepolymer curing agent $(10: 1)$ was poured over the mold and cured at $80^{\circ} \mathrm{C}$ for $1 \mathrm{~h}$. The mold was then peeled off, and holes were punched through the PDMS at the inlets and outlet of the channel.

The channel was covalently bonded to the glass chips containing the micropatterned gold electrodes by treating both the PDMS surface and the glass substrate with oxygen plasma at a radio frequency power of $30 \mathrm{~W}$ and a chamber pressure of $300 \mathrm{mTorr}$ for $1 \mathrm{~min}$. The resulting platform was heated at $80{ }^{\circ} \mathrm{C}$ for $1 \mathrm{~h}$ to help separating $\mathrm{H}_{2} \mathrm{O}$ molecules from the glass/PDMS interface during bond formation.

\subsection{Droplet generation and electrochemical conditions}

Droplets of vegetable oil (density of $0.91 \mathrm{~g} \mathrm{~cm}^{-3}$ and kinematic viscosity of $0.63 \mathrm{~cm}^{2} \mathrm{~s}^{-1}$ ) were generated at the T-junctions of the microfluidic device. The oil stream entered the crossflow junction perpendicularly to the electrolyte (sulphuric acid) stream, shearing off into discrete oil droplets. A surfactant (sodium dodecyl sulphate, 1\%) was added to the electrolyte to facilitate droplet formation and transport. Indeed, the surfactant acts to stabilize the interface between the two immiscible phases by minimizing the interfacial tension. Both oil and electrolyte were forced into the system with two syringe pumps (Razel R99-E). Teflon capillary tubes with inner diameter of 0.5 $\mathrm{mm}$ and outer diameter of $1.59 \mathrm{~mm}$ were used to connect the 5-mL syringes to the inlets of the microfluidic device. Droplets of various sizes were created according to the secondary channel used and the oil and electrolyte flow rates that varied up to $2 \mu \mathrm{L} / \mathrm{s}$.

A digital camera (Basler acA 1300-30uc) was used to film the passage of droplets close to the electrodes and determine their size and position for comparison with the results obtained from the ER variations. However, it was quite impossible to install the camera on the lateral side of the microfluidic device to measure both size and vertical position of the droplets so that only the droplet size was measured by the camera positioned above the microfluidic device. Moreover, to analyze 
the droplet images and the ER transients simultaneously recorded and be able to associate a given droplet image to the corresponding ER transient, only oil droplets of diameter larger than $50 \mu \mathrm{m}$ were generated since they flowed much more slowly in the detection channel than smaller droplets.

The continuous phase (electrolyte) was a $1 \mathrm{M} \mathrm{H}_{2} \mathrm{SO}_{4}$ solution prepared with deionised water. Its conductivity was measured before each experiment with a conductivity meter (Meterlab CDM230) and adjusted to the value of $45.5 \mathrm{~S} / \mathrm{m}$ by adding a few droplets of sulfuric acid or water. During the experiments, a pair of gold electrodes were polarized in the sulfuric acid solution by applying a $0 \mathrm{mV}$ potential difference between them with a home-made potentiostat. As a consequence, both electrodes were at their equilibrium potential and no DC current was flowing across them. In that conditions, the ER measurements were not influenced by mass- or chargetransfer at the electrodes. The other electrodes were disconnected and no electrode reference was used in this work.

\subsection{Technique for measuring ER fluctuations in microsystems}

The fluctuations of the ER between the two electrodes were measured using a home-made device [50,51] (Fig. 12). A sinusoidal voltage $v_{\mathrm{hf}}$ of amplitude $50 \mathrm{mV}$ and frequency $f_{100}=100 \mathrm{kHz}$ was superimposed on the applied voltage, which led to a current response of amplitude $v_{\mathrm{hf}} /\left|Z_{100}\right|$, where $\left|Z_{100}\right|$ is the modulus of the electrochemical impedance of the pair of electrodes. In contrast to electrochemical systems with large electrodes for which the impedance $Z_{100}$ is reduced to the ER, the surface area of the electrodes in microsystems is small and the effect of the electrochemical double layer cannot be ignored. In that case, the impedance at high-frequency, $Z_{\mathrm{HF}}(f)$, is reduced to $R_{\mathrm{e}}$ in series with the double layer capacitance $C_{\mathrm{dl}}: Z_{\mathrm{HF}}(f)=R_{\mathrm{e}}+1 / j C 2 \pi f$. The lower the electrode surface, the lower the time constant $R_{\mathrm{e}} C_{\mathrm{dl}}$, and the higher the absolute value of the phase angle at $100 \mathrm{kHz},|\varphi|=\arctan \left(1 / 2 \pi f_{100} R_{\mathrm{e}} C_{\mathrm{dl}}\right)$. As an example, Fig. 13 shows the Nyquist plot of the impedance of a pair of electrodes measured using a potentiostat Gamry Ref600 with an excitation signal of amplitude $10 \mathrm{mV}$ rms. It can be seen that the influence of the double layer capacitance is 
$\left.100=-77.2^{\circ}\right)$ and that measurements at frequencies above $1 \mathrm{MHz}$ are necessary to reach the ER. Since our home-made potentiostat was not able to work at such high frequencies, it was necessary to design a new method to measure the ER fluctuations using the applied voltage signal at $100 \mathrm{kHz}$.

The current $\Delta I+i_{\mathrm{hf}}$ flowing between the two electrodes was measured across the resistor $R$, amplified by a gain $G_{1}$ and the DC component of the voltage signal was removed by a capacitor (Fig. 12). After amplification by a gain $G_{2}$, the spontaneous current fluctuations, $\Delta I$, were eliminated with a high-pass filter cutting off at $17 \mathrm{kHz}$ and the sine wave signal was rectified by a diode and low-pass filtered at a cut-off frequency of $10 \mathrm{kHz}$. The DC component of the output signal, $v_{|Z|_{100}}$, was measured and its fluctuations were acquired, after DC-offset removal, amplification with a gain $G_{3}=-10$, and analog low-pass filtering to prevent aliasing, with a realtime data acquisition system (Concurrent Computer, Maxion 9100) at different sampling frequencies. The output voltage signal, $v_{|Z|_{100}}$, obeys the following expression:

$$
v_{|Z|_{100}}=b \frac{1}{|Z|_{100}}+d
$$

in which the parameters $b$ and $d$ could be determined through a calibration procedure consisting in replacing the electrochemical cell by pure resistors. From Eq. 9, the mean value of $|Z|_{100}$ could be calculated from the mean value of $v_{|Z|_{100}}$ measured during the electrochemical experiments and the variations of $|Z|_{100}$ could be derived from the measured variations $\Delta v_{|Z|_{100}}$ with the equation:

$$
\Delta|Z|_{100}=-\frac{\left(\left.Z\right|_{100}\right)^{2}}{b G_{3}} \Delta v_{\text {meas }}
$$

obtained by differentiating Eq. 9 for $|Z|_{100}$ variations of small amplitude.

The last step in the measurement procedure is the calculation of the ER variations, $\Delta R_{\mathrm{e}}$, from the measured variations of $\Delta|Z|_{100}$. As shown in Fig. 13, an increase in the ER corresponds to a shift of the impedance at $100 \mathrm{kHz}$ from point A to point A'. For small ER variations compared to 
the impedance modulus at $100 \mathrm{kHz}$, the phase angle $\varphi_{100}$ varies very little so that the segment $\mathrm{AB}$ can be considered as orthogonal to BA', the length of which is equal to $\Delta|Z|_{100}$. As a consequence, it is straightforward to write $\Delta R_{\mathrm{e}}$ as a function of $\Delta|Z|_{100}$ :

$$
\Delta R_{\mathrm{e}}=\frac{\Delta|Z|_{100}}{\cos \varphi_{100}}
$$

In order to validate the measurement procedure, experiments with a dummy cell (Fig. 14) showing the same behaviour at high frequency were performed. The dummy cell consisted of a resistance $R_{\mathrm{e}}=500 \Omega$, in series with a resistance $R_{\mathrm{t}}=700 \mathrm{k} \Omega$ in parallel with a capacitance $C_{\mathrm{dl}}=$ $1.2 \mathrm{nF} . R_{\mathrm{e}}$ represents the electrolyte resistance, $R_{\mathrm{t}}$ the charge transfer resistance and $C_{\mathrm{dl}}$ the double layer capacitance. To simulate a change in ER, another resistance, $r_{\mathrm{e}}=200 \Omega$, and an electronic switch (Littlefuse Inc., HE 3621 Q0510) in parallel, were added in series with $R_{\mathrm{e}}$. The switch was connected to a square signal generator to control its periodic opening and closing (frequency of 10.2 $\mathrm{Hz}$ ) so that the high-frequency resistance of the dummy cell changed periodically between $R_{\mathrm{e}}$ and $R_{\mathrm{e}}+r_{\mathrm{e}}$. It should be noted that the value of $r_{\mathrm{e}}$ represents a large fluctuation of ER (40\%) but a relatively small change compared to $|Z|_{100}(14.3 \%)$. Fig. $14 \mathrm{~b}$ shows the high-frequency part of the impedance of the dummy cell measured when the switch was closed. At $100 \mathrm{kHz}$, the modulus and phase are equal to $1398 \Omega$ and $68.47^{\circ}\left(\cos \varphi_{100}=0.367\right)$, respectively. The ER measurements were then performed when the switch was automatically turned on and off. Fig. 15a shows the periodic change with time of $|Z|_{100}$ calculated from the measured $v_{|Z|_{100}}$ signal with Eq. 10 (the base value of $500 \Omega$ has been eliminated for a clearer reading of the figure) and Fig. $15 \mathrm{~b}$ presents the corresponding $\Delta R_{\mathrm{e}}$ variations derived with Eq. 11. If the over-oscillations due to the presence of the analog anti-aliasing filter are ignored, the amplitude of the $|Z|_{100}$ steps is $73.45 \Omega$, which gives a $\Delta R_{\mathrm{e}}$ value of $200.14 \Omega$ very close to the $r_{\mathrm{e}}$ value. The measurement procedure could then be considered valid for measuring the ER fluctuations due to the passage of oil droplets in the microchannel. 


\section{Results and discussion}

Fig. 16 shows the image of an oil droplet of $86 \mu \mathrm{m}$ in diameter before its passage in front of the electrodes (dark ribbons) and the corresponding experimental $R_{\mathrm{e}}(t)$ transient (black curve). The theoretical (red curve) fitted with Eq. 8 by replacing $x$ with $v\left(t-t_{0}\right)$, where $v$ is the velocity of the droplet and $t_{0}$ the time instant at which the sphere passes at the position $x=0$, is given as well. The excellent agreement between the experimental and theoretical curves is an indication that the droplet flowed horizontally close to the centre of the channel $(z \approx 0)$. Moreover, transients with a flat top as in Fig. 4, indicating that the droplet approached very near to the electrodes $(h<1 \mu \mathrm{m})$, were never measured. From the adjusted parameters $\left(t_{0}=23.843 \mathrm{~s}, d_{\mathrm{p}}=86.51 \mu \mathrm{m}\right.$, and $v=279.1$ $\mu \mathrm{m} \mathrm{s}^{-1}$ ), it can be concluded that the estimation of the droplet diameter from the ER variations is precise (error of $0.6 \%$ ). The droplet velocity, which could be assumed to be constant in front of the electrodes because of the perfect symmetry of the $R_{\mathrm{e}}$ transients, could also be obtained from the ER measurements. This allows the Reynolds number of the hydrodynamic flow to be derived. The following equation:

$$
R e=\frac{\rho_{\mathrm{e}} d_{\mathrm{p}} v}{\eta}
$$

where $\rho_{e}$ and $\eta$ are the density and dynamic viscosity of the electrolyte, respectively $\left(\rho_{e}=1.065\right.$ $\mathrm{g} \mathrm{cm}^{-3}$ and $\left.\eta=1.0 \mathrm{~g} \mathrm{~m}^{-1} \mathrm{~s}^{-1}\right)$, gives a low Reynolds number $(\operatorname{Re}=0.026)$, as usual for fluid flows in microchannels.

Experiments with various droplet sizes were performed successively. Fig. 17 shows a comparison of the droplet diameters estimated by image analysis and ER measurements. The diameter of the droplet was calculated as the average of 12 values measured in different radial directions on the droplet image. The slope close to one with a deviation of $0.39 \mu \mathrm{m}$ from the $x$-axis origin confirms the good agreement between the two techniques. Large oil droplets were generated preferably in this work because they were slower than small droplets and it was then easier to correlate the images of the droplet to the corresponding ER transient. Nevertheless, the EN 
technique is able to detect smaller droplets. Indeed, the standard deviation of the background noise in Fig. 16 is $1.9 \Omega$, and a careful examination of the figure shows that the noise was in great part due to the mains (50 Hz and harmonics). Digital low-pass filtering of the signal to avoid mains interference allows the standard deviation to be decreased down to $0.74 \Omega$ (this could have also been obtained by improving the shielding of the experimental set-up). This value corresponds to a droplet diameter of $17 \mu \mathrm{m}$, so, roughly speaking, only droplets of diameter larger than $20-25 \mu \mathrm{m}$ can be detected with the microdevice used. Detection and sizing of smaller droplets require using a microchannel and electrodes of smaller dimensions.

Considering the duration time of about $1 \mathrm{~s}$ for the ER transient shown in Fig. 16b, the droplet frequency that can be detected is close to $1 \mathrm{~Hz}$, which is much lower than that obtained by optical techniques (typically $1 \mathrm{kHz}$ ). However, the detection frequency can be increased by several orders of magnitude. Indeed, the experimental set-up in this work is based on the use of a sinusoidal signal at $100 \mathrm{kHz}$ so that the maximum droplet frequency the system can detect is given by the maximum sampling frequency (presently $15 \mathrm{kHz}$ ) and the number of data points employed to correctly define the shape of the electrolyte resistance transients due to the passage of single droplets. For example, 10 points give a transient duration of $0.67 \mathrm{~ms}$ and a maximum droplet frequency of $1.5 \mathrm{kHz}$, which may still be lower than that obtained with specific optical techniques, but with the advantage for the present method to be operable in opaque microfluidic devices. Of course, higher droplet frequency could be obtained by increasing the frequency of the sinusoidal signal. However, such high values of droplet detection frequency cannot be obtained using the present microdevice since a limitation comes from the flow rate in the channel. According to Figs. 3, 4 , and 9 , the length of the detection region is roughly equal to $D+2 d_{\mathrm{x}}$ so that the distance $L$ between two successive droplets must be higher than $D+2 d_{\mathrm{x}}$ for a correct analysis of single droplets. As a consequence, the droplet generation frequency, $f_{\mathrm{g}}$, is lower than $v /\left(D+2 d_{\mathrm{x}}\right)$, where $v$ is the droplet velocity. A $f_{\mathrm{g}}$ value of $1 \mathrm{kHz}$ would necessitate a fast droplet velocity of $30 \mathrm{~cm} \mathrm{~s}^{-1}$ (corresponding to a Reynolds number of 16 for an oil droplet of $50 \mu \mathrm{m}$ in diameter) that cannot be 
obtained in microchannels. However, limiting the droplet velocity to $1 \mathrm{~cm} \mathrm{~s}^{-1}$ and decreasing the length of the detection region to $10 \mu \mathrm{m}$ would lead to a droplet generation frequency of $1 \mathrm{kHz}$ and the single droplets generated could be detected with the present equipment.

Plug-like oil droplets were also characterized with the EN technique. Fig. 18 shows the variations of the ER due to the quasi-periodic passage of oil plugs above the electrodes. Since the length, $L_{\mathrm{p}}$, of the plugs was larger than the detection region, the ER time record shows a succession of step-like transients with a constant maximum value when both electrodes were completely screened by a plug. In that case, the current was flowing across the thin film of electrolyte adhering to the walls of the microdevice [57]. A rough estimate of the plug velocity can be derived in two ways from the analysis of the durations, $t_{\text {up }}$ and $t_{\text {down }}$, of the upper and lower parts of the ER transient respectively, by assuming a cuboid shape of dimensions $L_{\mathrm{p}}, l_{\mathrm{y}}, l_{\mathrm{z}}$ for the plug. Indeed, if it is assumed that the front of the plug corresponds exactly to the right side of the right electrode at the beginning of the transient and that the back of the plug corresponds to the left side of the left electrode at the end of the transient, the distance traveled by the plug during the time $t_{\text {up }}$ of the transient is equal to $L_{\mathrm{p}}-D-2 d_{\mathrm{x}}$, so that the plug velocity is given by:

$$
v_{\mathrm{p}}=\frac{L_{\mathrm{p}}-D-2 d_{\mathrm{x}}}{t_{\mathrm{up}}}
$$

Under the same assumptions, if $D_{\mathrm{p}}$ denotes the distance between two successive plugs $\mathrm{A}$ and B (see Fig. 18b), the distance traveled by plug B between the beginning of transient $\mathrm{A}$ and the beginning of transient $\mathrm{B}$, that is during the time $t_{\mathrm{up}}+t_{\text {down }}$, is equal to $D_{\mathrm{p}}+L_{\mathrm{p}}$, which gives a plug velocity of:

$$
v_{\mathrm{p}}=\frac{D_{\mathrm{p}}+L_{\mathrm{p}}}{t_{\mathrm{up}}+t_{\mathrm{down}}}
$$

Consistently, from the values estimated from Fig. $18\left(L_{\mathrm{p}}=520 \mu \mathrm{m}, D_{\mathrm{p}}=490 \mu \mathrm{m}, t_{\mathrm{up}}=180\right.$ $\mathrm{ms}$, and $\left.t_{\text {down }}=600 \mathrm{~ms}\right)$, the values of the plug velocity given by Eqs. 13 and 14 are very close $\left(v_{\mathrm{p}}=\right.$ $1.2 \mathrm{~mm} \mathrm{~s}^{-1}$ and $\left.1.3 \mathrm{~mm} \mathrm{~s}^{-1}\right)$. It should be noted that the characteristic parameters of the oil plug $\left(L_{\mathrm{p}}\right.$ 
and $D_{\mathrm{p}}$ ) could be estimated from Eqs. 13 and 14 if the plug velocity was previously measured, which could be done by working with a second pair of electrodes and measuring the time lag between two ER transients corresponding to the passage of a given plug above each pair of electrodes.

It is important to mention that the ER transients are influenced by the presence of more than one droplet when the distance between two successive droplets is smaller than the detection region. In that case, droplet sizing is not possible from the ER time records and signal analysis has to be performed in the frequency domain by measuring the power spectral density of the ER signal to determine the frequency of passing droplets. Otherwise, another possibility consists in decreasing the width of the detection region with smaller electrodes and interelectrode distance.

As shown in this paper, detection and sizing of single droplets passing above the measuring electrodes is possible in a conductive medium. Detection of aqueous droplets in a low-conductive carrier solution is more involved since it relies on the modification of the current lines between the electrodes by the passing droplets. In that case the current lines are located very close to the electrodes so that it should be difficult to detect water droplets passing far above the electrodes. However, chronoamperometry [30-32] or impedance [39-40] measurements have already been performed to study water-in-oil droplets in microchannels. In that case, long oblate droplets or plugs covering the electrodes were used, so that variations of current or resistance could be measured. Obviously, the size and the distance between the electrodes must be adapted to the size of the aqueous droplets investigated.

\section{Conclusions}

This work investigates the possibility to use a specific electrochemical noise technique consisting in measuring the electrolyte resistance between two immerged electrodes to detect, track and size small particles circulating in a microfluidic channel. The main advantage of this technique is its ability to characterize microparticles precisely without the help of optical techniques that are 
often used in lab-on-a-chip devices. Even if not shown in this paper, the EN technique can measure ER variations in the millisecond range for the detection of fast particles.

The influence of the size and position of the particle, as well as the dimension of the electrodes and the interelectrode distance, on the ER was calculated with a theoretical model using the finite element method. The ER measurements performed with spherical oil droplets circulating in the microchannel show an excellent agreement with the theoretical predicted ER values. The length of oil plugs and the distance between two successive plugs could also been determined from the ER variations, their values being in good concordance with those derived from images of the flow above the electrodes.

Single-particle sizing or particle counting is then possible with the EN technique in opaque microfluidic devices. For the microchannel used (characteristic dimensions of $100 \mu \mathrm{m}$ ), particles of diameter larger than 20-25 $\mu \mathrm{m}$ could be characterized with an error of a few percents that can be decreased if the droplets are forced to flow in the middle of the channel $(z \approx 0)$. Developments are in progress to characterize smaller particles, such as biological cells for example, which requires decreasing the size of the microchannel and the electrodes, and increasing the frequency at which the ER variations are measured.

\section{Acknowledgements}

The authors would like to acknowledge the staff of the IEF (Institut d'Electronique Fondamentale, Université Paris Sud, CNRS) cleanroom and L. Becerra from INSP (Institut des NanoSciences de Paris, Université Pierre et Marie Curie, UPMC-CNRS) for fruitful discussions and their help in the fabrication of the microfluidic device. M. Turmine and V. Vivier in LISE laboratory are also thanked for their help in this work.

\section{Biographies}


Nour Eddin Yakdi obtained his PhD degree in Physical Chemistry in 2015 from the University Pierre and Marie Curie (UPMC) in Paris, France. His research is focused on micro and nanofabrication as well as microfluidics. During his $\mathrm{PhD}$, he used the electrochemical noise technique for the characterization of microparticles flowing in a lab-on-a-chip device. He is now with the Fluigent company.

François Huet received his $\mathrm{PhD}$ degree in Physical Sciences in 1984 from the University Pierre and Marie Curie (UPMC) in Paris, France. He worked at the Centre National de la Recherche Scientifique (CNRS) until 2006 when he was named as full professor at UPMC in 2006. He is Head of the Laboratory Interfaces and Electrochemical Systems (LISE) since 2009. His research is focused on the development of the electrochemical noise technique for applications in corrosion and two-phase systems and on the use of the electrochemical impedance technique in the field of batteries.

Kieu Ngo obtained his PhD in Micro and Nanoelectronics in 2006 from the Paul Cézanne University (Marseille, France). He is now assistant professor at the Pierre and Marie Curie University in Paris (France). His current research interests focus on droplet-based microfluidics and electrochemical techniques. 


\section{References}

[1] G. M. Whitesides, The origins and the future of microfluidics, Nature 442 (2006) 368-373.

[2] P. N. Nge, C. I. Rogers and A. T. Woolley, Advances in microfluidic materials, functions, integration, and applications, Chem. Rev. 113 (2013) 2550-2583.

[3] E. K. Sackmann, A. L. Fulton and D. J. Beebe, The present and future role of microfluidics in biomedical research, Nature 507 (2014) 181-189.

[4] K. S. Elvira, X. Casadevall i Solvas, R. C. R. Wootton and A. J. deMello, The past, present and potential for microfluidic reactor technology in chemical synthesis, Nat. Chem. 5 (2013) 905-915.

[5] J. Zhang, S. Yan, D. Yuan, G. Alici, N.-T. Nguyen, M. E. Warkiani and W. Li, Fundamentals and applications of inertial microfluidics: a review, Lab Chip 16 (2016) 10-34.

[6] H. Song, J. D. Tice and R. F. Ismagilov, A microfluidic system for controlling reaction networks in time, Angew. Chem. Int. Ed. 42 (2003) 767-772.

[7] H. Song, D. L. Chen and R. F. Ismagilov, Reactions in droplets in microfluidic channels, Angew. Chem. Int. Ed. 45 (2006) 7336-7356.

[8] A. B. Theberge, F. Courtois, Y. Schaerli, M. Fischlechner, C. Abell, F. Hollfelder and W. T. S. Huck, Microdroplets in microfluidics: an evolving platform for discoveries in chemistry and biology, Angew. Chem. Int. Ed. 49 (2010) 5846-5868.

[9] S.-Y. Teh, R. Lin, L.-H. Hung and A. P. Lee, Droplet microfluidics, Lab Chip 8 (2008) 198220.

[10] X. Casadevall i. Solvas and A. deMello, Droplet microfluidics: recent developments and future applications, Chem. Commun. 47 (2011) 1936-1942.

[11] R. Seemann, M. Brinkmann, T. Pfohl and S. Herminghaus, Droplet based microfluidics, Rep. Prog. Phys. 75 (2012) 016601-016641.

[12] C. N. Baroud, F. Gallaire and R. Dangla, Dynamics of microfluidic droplets, Lab Chip 10 (2010) 2032-2045. 
[13] H. Gu, M. H. G. Duits and F. Mugele, Droplets formation and merging in two-phase flow microfluidics, Int. J. Mol. Sci. 12 (2011) 2572-2597.

[14] S.-Y. Park, T.-H. Wu, Y. Chen, M. A. Teitell and P.-Y. Chiou, High-speed droplet generation on demand driven by pulse laser-induced cavitation, Lab Chip 11 (2011) 1010-1012.

[15] H. N. Joensson and H. Andersson-Svahn, Droplet Microfluidics-A tool for single-cell analysis, Angew. Chem. Int. Ed. 51 (2012) 12176-12192.

[16] T. P. Lagus and J. F. Edd, A review of the theory, methods and recent applications of highthroughput single-cell droplet microfluidics, J. Phys. D: Appl. Phys. 46 (2013) 114005.

[17] A. Rakszewska, J. Tel, V. Chokkalingam and W. T. S. Huck, One drop at a time: toward droplet microfluidics as a versatile tool for single-cell analysis, NPG Asia Mater. 6 (2014) e133.

[18] S. Seiffert, Small but smart: sensitive microgel capsule, Angew. Chem. Int. Ed. 52 (2013) 11462-11468.

[19] R. Riahi, A. Tamayol, S. A. M. Shaegh, A. M. Ghaemmaghami, M. R. Dokmeci and A. Khademhosseini, Microfluidics for advanced drug delivery systems, Curr. Opin. Chem. Eng. 7 (2015) 101-112.

[20] K. V. I. S. Kaler and R. Prakash, Droplet microfluidics for chip-based diagnostics, Sensors 14 (2014) 23283-23306.

[21] S. Tasoglu, U. A. Gurkan, S. Q. Wang and U. Demirci, Manipulating biological agents and cells in micro-scale volumes for applications in medicine, Chem. Soc. Rev. 42 (2013) 57885808.

[22] N.-T. Nguyen, S. Lassemono and F. A. Chollet, Optical detection for droplet size control in microfluidic droplet-based analysis systems, Sensor. Actuat. B 117 (2006) 431-436.

[23] M. Srisa-Art, A. J. deMello and J. B. Edel, High-efficiency single-molecule detection within trapped aqueous microdroplets, J. Phys. Chem. B 114 (2010) 15766-15772. 
[24] D. Bardin and A. P. Lee, Low-cost experimentation for the study of droplet microfluidics, Lab Chip 14 (2014) 3978-3986.

[25] Z. Li, A. M. Leshansky, L. M. Pismen and P. Tabeling, Step-emulsification in a microfluidic device, Lab Chip 15 (2015) 1023-1031.

[26] M. Kim, M. Pan, Y. Gai, S. Pang, C. Han, C. Yang and S. K. Y. Tang, Optofluidic ultrahighthroughput detection of fluorescent drops, Lab Chip 15 (2015) 1417-1423.

[27] Y. H. Ghallab and W. Badawy, in Lab-on-a-chip: techniques, circuits, and biomedical applications, Artech House, Norwood, MA, USA, 2010, Chapter 5, pp. 63-76.

[28] Y. Zhu and Q. Fang, Analytical detection techniques for droplet microfluidics-A review, Anal. Chim. Acta 787 (2013) 24-35.

[29] N. V. Rees, S. M. Matthews, K. Yunus, A. C. Fisher and R. G. Compton, A method for the positioning and tracking of small moving particles, Angew. Chem. Int. Ed. 48 (2009) 23762378.

[30] H. Liu and R. M. Crooks, Highly reproducible chronoamperometric analysis in microdroplets, Lab Chip 13 (2013) 1364-1370.

[31] X. Lin, X. Hu, Z. Bai, Q. He, H. Chen, Y. Yan and Z. Ding, A microfluidic chip capable of switching W/O droplets to vertical laminar flow for electrochemical detection of droplet contents, Anal. Chim. Acta 828 (2014) 70-79.

[32] A. Suea-Ngam, P. Rattanarat, O. Chailapakul and M. Srisa-Art, Electrochemical droplet-based microfluidics using chip-based carbon paste electrodes for high-throughput analysis in pharmaceutical applications, Anal. Chim. Acta 883 (2015) 45-54.

[33] L. L. Sohn, O. A. Saleh, G. R. Facer, A. J. Beavis, R. S. Allan and D. A. Notterman, Capacitance cytometry: Measuring biological cells one by one, Proc. Natl. Acad. Sci. USA 97 (2000) 10687-10690. 
[34] S. Murali, X. Xia, A. V Jagtiani, J. Carletta and J. Zhe, Capacitive Coulter counting: detection of metal wear particles in lubricant using a microfluidic device, Smart Mater. Struct. 18 (2009) 037001.

[35] C. Elbuken, T. Glawdel, D. Chan and C. L. Ren, Detection of microdroplet size and speed using capacitive sensors, Sensor. Actuat. A 171 (2011) 55-62.

[36] T. Sun and H. Morgan, Single-cell microfluidic impedance cytometry: a review, Microfluid. Nanofluid. 8 (2010) 423-443.

[37] S. Gawad, L. Schild and Ph. Renaud, Micromachined impedance spectroscopy flow cytometer for cell analysis and particle sizing, Lab Chip 1 (2001) 76-82.

[38] S. Gawad, K. Cheung, U. Seger, A. Bertsch and Ph. Renaud, Dielectric spectroscopy in a micromachined flow cytometer: theoretical and practical considerations, Lab Chip 4 (2004) $241-251$.

[39] C. Luo, X. Yang, Q. Fu, M. Sun, Q. Ouyang, Y. Chen and H. Ji, Picoliter-volume aqueous droplets in oil: Electrochemical detection and yeast cell electroporation, Electrophoresis 27 (2006) 1977-1983.

[40] E. W. M. Kemna, L. I. Segerink, F. Wolbers, I. Vermes and A. van den Berg, Label-free, high-throughput, electrical detection of cells in droplets, Analyst 138 (2013) 4585-4592.

[41] R. Rodriguez-Trujillo, M. A. Ajine, A. Orzan, M. D. Mar, F. Larsen, C. H. Clausen and W. E. Svendsen, Label-free protein detection using a microfluidic Coulter-counter device, Sensor. Actuat. B 190 (2014) 922-927.

[42] J. Mok, M. N. Mindrinos, R. W. Davis and M. Javanmard, Digital microfluidic assay for protein detection, Proc. Natl. Acad. Sci. USA 111 (2014) 2110-2115.

[43] S. Gawad, T. Sun, N. G. Green and H. Morgan, Impedance spectroscopy using maximum length sequences: Application to single cell analysis, Rev. Sci. Instrum. 78 (2007) 054301.

[44] K. Cheung, S. Gawad and Ph. Renaud, Impedance spectroscopy flow cytometry, Cytom. Part A 65 A (2005) 124-132. 
[45] C. Gabrielli, F. Huet, R. Wiart and J. Zoppas-Ferreira, Dynamic behaviour of an electrolyser with a two phase solid-liquid electrolyte. Part II: Investigation of elementary phenomena and electrode modelling, J. Appl. Electrochem. 24 (1994) 1235-1243.

[46] V. Bouet, J. Fransaer, F. Huet, G. Maurin and J.P. Célis, Electrochemical resistance noise during composite plating, J. Electrochem. Soc. 145 (1998) 436-446.

[47] H. Bouazaze, F. Huet and R. P. Nogueira, A new approach for monitoring corrosion and flow characteristics in oil/brine mixtures, Electrochim. Acta 50 (2005) 2081-2090.

[48] C. Gabrielli, F. Huet, M. Keddam and A. Sahar, Investigation of water electrolysis by spectral analysis. I. Influence of the current density, J. Appl. Electrochem. 19 (1989) 683-696.

[49] J. M. Malo, J. Uruchurtu and O. Corona, Corrosion detection of mild steel in a two-phase hydrocarbon/electrolyte system under flow conditions using electrochemical noise, Corrosion 58 (2002) 932-940.

[50] C. Gabrielli, F. Huet and M. Keddam, Real-time measurement of electrolyte resistance fluctuations, J. Electrochem. Soc. 138 (1991) L82-L84.

[51] N. Yakdi, F. Huet and K. Ngo, In-situ particle sizing at millimeter scale from electrochemical noise: simulation and experiments, Electrochim. Acta 180 (2015) 1050-1058.

[52] H. Bouazaze, J. Fransaer, F. Huet, P. Rousseau and V. Vivier, Electrolyte-resistance change due to an insulating sphere in contact with a disk electrode, Electrochim. Acta 55 (2010) $1645-1655$.

[53] A. T. Tran, F. Huet, K. Ngo and P. Rousseau, Influence on the electrolyte resistance of the contact angle of a bubble attached to a disk electrode, J. Electroanal. Chem. 737 (2015) 114122.

[54] P. J. Sides and C. W. Tobias, Primary potential and current distribution around a bubble on an electrode, J. Electrochem. Soc. 127 (1980) 288-291.

[55] J. Fransaer, J. P. Celis and J. R. Roos, Variations in the flow of current to disk electrodes caused by particles. J. Electroanal. Chem. 391 (1995) 11-28. 
[56] R. W. DeBlois and C. P. Bean, Counting and Sizing of Submicron Particles by the Resistive Pulse Technique, Rev. Sci. Instrum. 41 (1970) 909-916.

[57] C. N. Baroud, F. Gallaire and R. Dangla, Dynamics of microfluidique droplets, Lab Chip 10 (2010) 2032-2045. 


\section{Figure captions}

Fig. 1 Geometry of the model. (a) channel and electrodes: $l_{\mathrm{x}}, l_{\mathrm{y}}, l_{\mathrm{z}}$ dimensions of the electrolyte volume, $D$ interelectrode distance, $d_{\mathrm{x}}$ length of the electrodes in the $x$ direction, (b) sphere of diameter $d_{\mathrm{p}}$ and position $h$ above the channel floor.

Fig. 2 Distribution of the current lines in the $\mathrm{Oxz}$ plane in the presence of an insulating sphere (blue disk) between the two electrodes $(x=y=0, z=0, \kappa=45.5 \mathrm{~S} / \mathrm{m})$.

Fig. 3 Variations of $R_{\mathrm{e}}$ and $\Delta R_{\mathrm{e}}$ as a function of the position of the insulating sphere along the flow direction $\mathrm{O} x$ and its vertical position $z\left(y=0, d_{\mathrm{p}}=50 \mu \mathrm{m}\right)$.

Fig. 4 Variations of $R_{\mathrm{e}}$ and $\Delta R_{\mathrm{e}}$ as a function of the position of the insulating sphere along the flow direction $\mathrm{O} x$ and its vertical position $z\left(y=0, d_{\mathrm{p}}=85 \mu \mathrm{m}\right)$.

Fig. 5 Variations of $R_{\mathrm{e}}$ and $\Delta R_{\mathrm{e}}$ as a function of the position of the insulating sphere along the $y$ axis $\left(x=0, z=0, d_{\mathrm{p}}=50 \mu \mathrm{m}\right)$.

Fig. 6 Variations of $\Delta R_{\mathrm{e}}$ and $\Delta R_{\mathrm{e}, \text { norm }}$ as a function of the diameter of the insulating sphere located at the centre of the channel $(x=y=z=0)$.

Fig. 7 Variations of $\Delta R_{\mathrm{e}, \max }$ and $\Delta R_{\mathrm{e}, \text { norm }}$ as a function of the vertical position and diameter of the insulating sphere $(x=y=0)$.

Fig. 8 Estimation of the sphere diameter from the measured value of $\Delta R_{\mathrm{e}, \max }(x=y=0)$.

Fig. 9 Values of $\Delta R_{\mathrm{e}}$ as a function of the position of the insulating sphere along the flow direction Ox for $y=z=0$ and different sphere diameters. Solid red lines obey Eq. 8 .

Fig. 10 Variations of $R_{\mathrm{e}}$ and $\Delta R_{\mathrm{e}}$ as a function of the conductivity of a sphere of diameter $50 \mu \mathrm{m}$ located at the centre of the channel $(x=y=z=0)$.

Fig. 11 Schematic illustration of the microfluidic device.

Fig. 12 Experimental setup used for measuring the ER fluctuations. 
Fig. 13 (a) High-frequency impedance Nyquist diagram of a pair of electrodes $\left(d_{\mathrm{x}}=D=100 \mu \mathrm{m}\right)$ and (b) method for measuring the $\Delta R_{\mathrm{e}}$ fluctuations.

Fig. 14 Dummy cell $\left(R_{\mathrm{e}}=500 \Omega, r_{\mathrm{e}}=200 \Omega, R_{\mathrm{t}}=700 \mathrm{k} \Omega, C_{\mathrm{dl}}=1.2 \mathrm{nF}\right)$ used for for validating the $\Delta R_{\mathrm{e}}$ measurements (a) and corresponding high-frequency impedance Nyquist diagram (b).

Fig. 15 Variations of $|Z|_{100}$ measured on the dummy cell in Fig. 14a (a) and corresponding variations of $R_{\mathrm{e}}(\mathrm{b})$.

Fig. 16 Image of an oil droplet of $86 \mu \mathrm{m}$ in diameter flowing in the microchannel (a) and corresponding experimental (black) and theoretical (red) $R_{\mathrm{e}}$ variations calculated with Eq. 9 and the fitted parameters $d_{\mathrm{p}}=86.51 \mu \mathrm{m}, t_{0}=23.843 \mathrm{~s}$, and $v=279.1 \mu \mathrm{m} \mathrm{s}^{-1}$ (b).

Fig. 17 Droplet diameter estimated from $\Delta R_{\mathrm{e}}$ variations as a function of the droplet diameter estimated from the images.

Fig. 18 Images of the passage of oil plugs above the L (left) and R (right) electrodes (a, b) and corresponding $\Delta R_{\mathrm{e}}$ variations (c). 


\section{Figures}
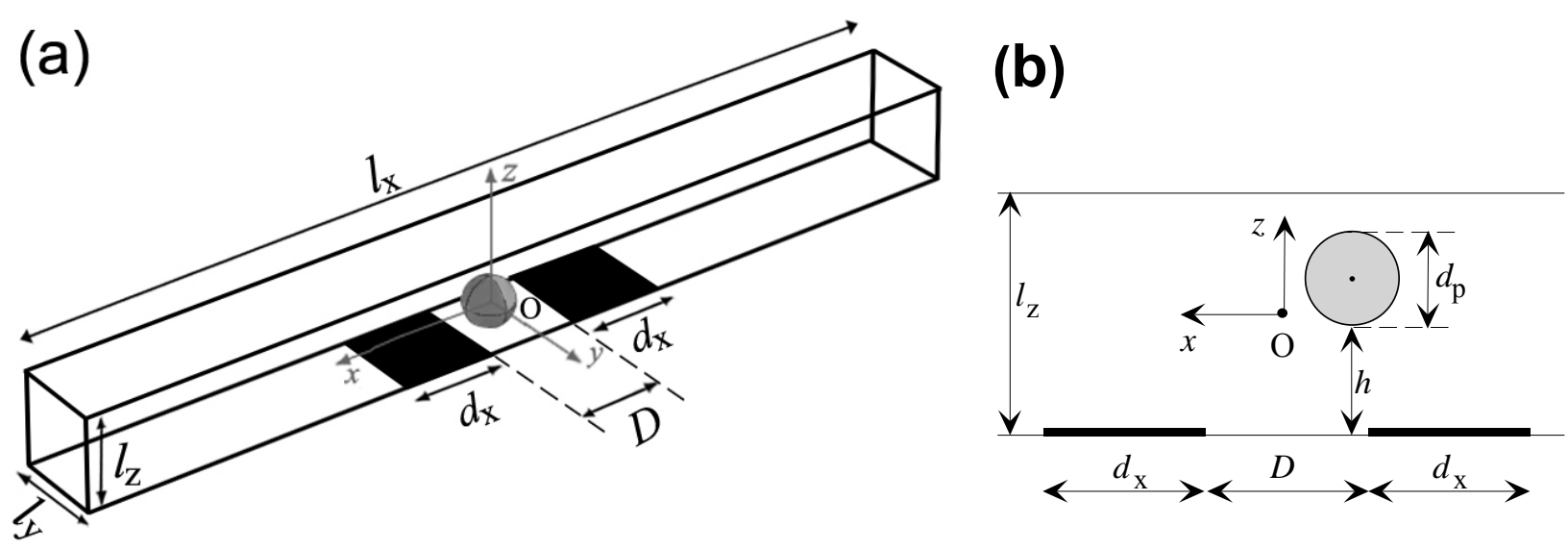

Fig. 1 Geometry of the model. (a) channel and electrodes: $l_{\mathrm{x}}, l_{\mathrm{y}}, l_{\mathrm{z}}$ dimensions of the electrolyte volume, $D$ interelectrode distance, $d_{\mathrm{x}}$ length of the electrodes in the $x$ direction, (b) sphere of diameter $d_{\mathrm{p}}$ and position $h$ above the channel floor. 


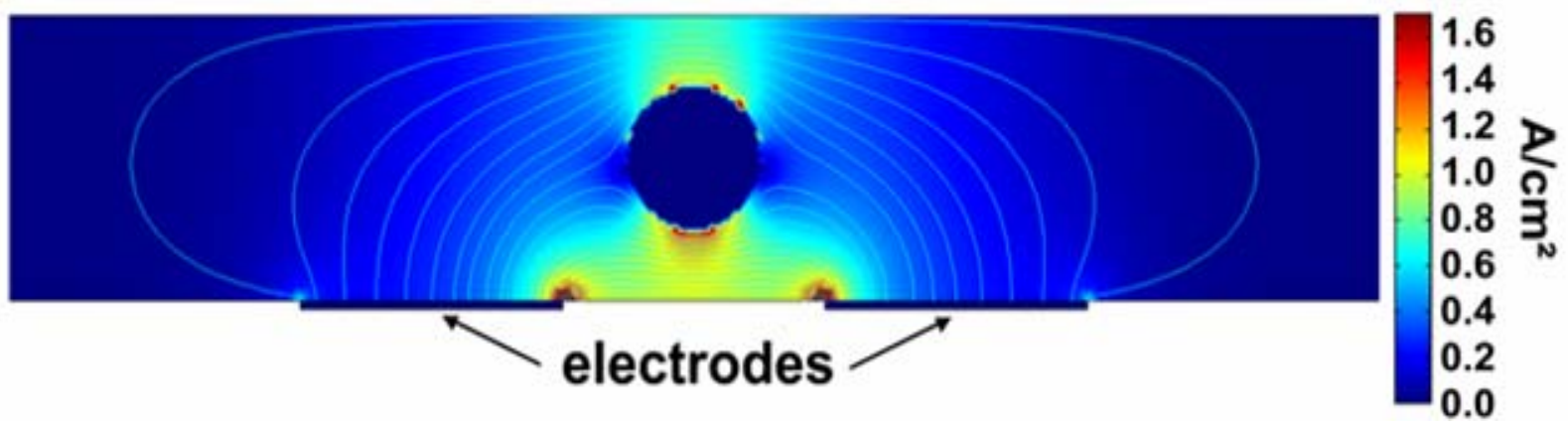

Fig. 2 Distribution of the current lines in the $\mathrm{Oxz}$ plane in the presence of an insulating sphere (blue disk) between the two electrodes $\left(x=y=0, \mathrm{z}=0, d_{\mathrm{p}}=50 \mu \mathrm{m}, \kappa=45.5 \mathrm{~S} / \mathrm{m}\right)$. 


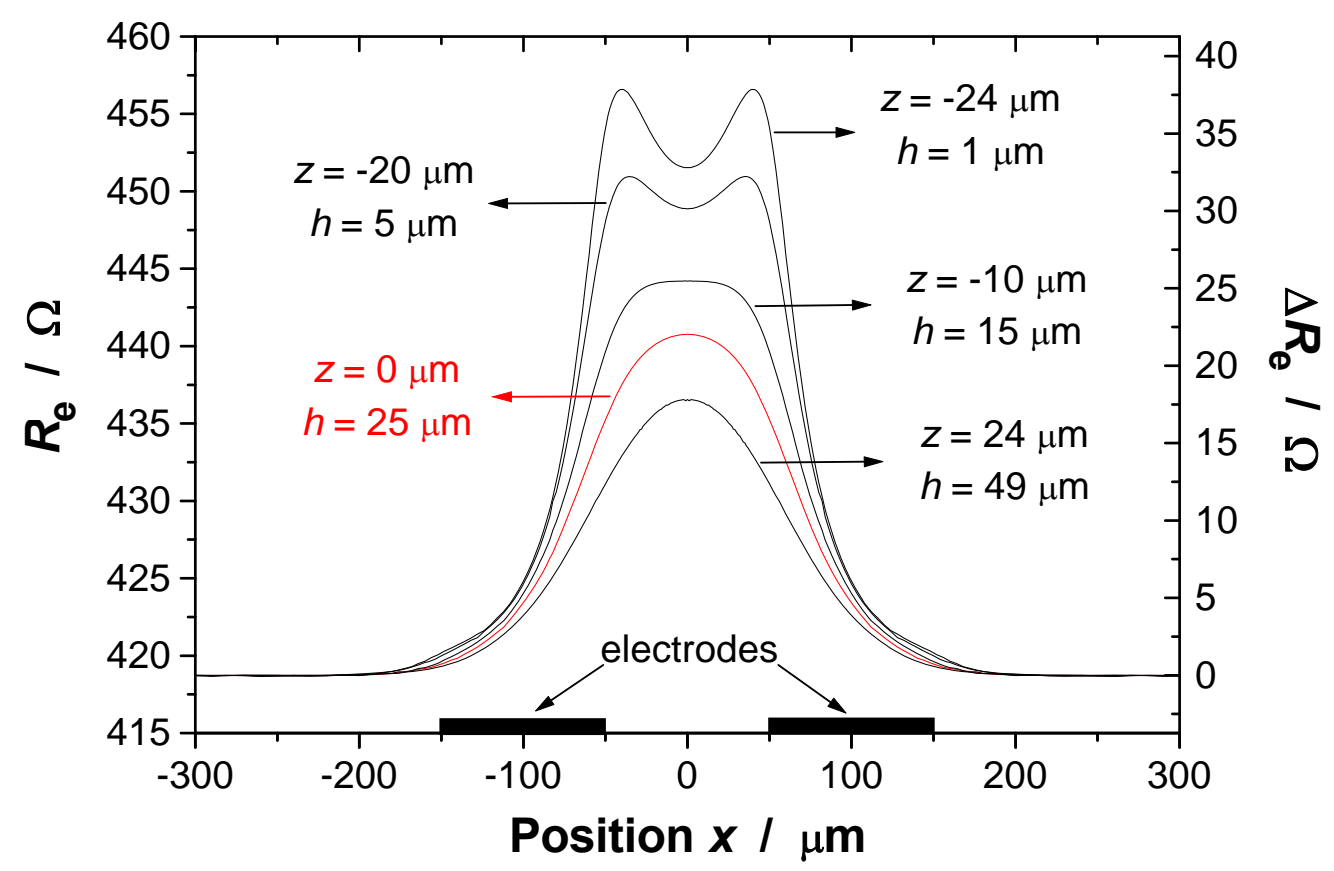

Fig. 3 Variations of $R_{\mathrm{e}}$ and $\Delta R_{\mathrm{e}}$ as a function of the position of the insulating sphere along the flow direction $\mathrm{O} x$ and its vertical position $z\left(y=0, d_{\mathrm{p}}=50 \mu \mathrm{m}\right)$. 


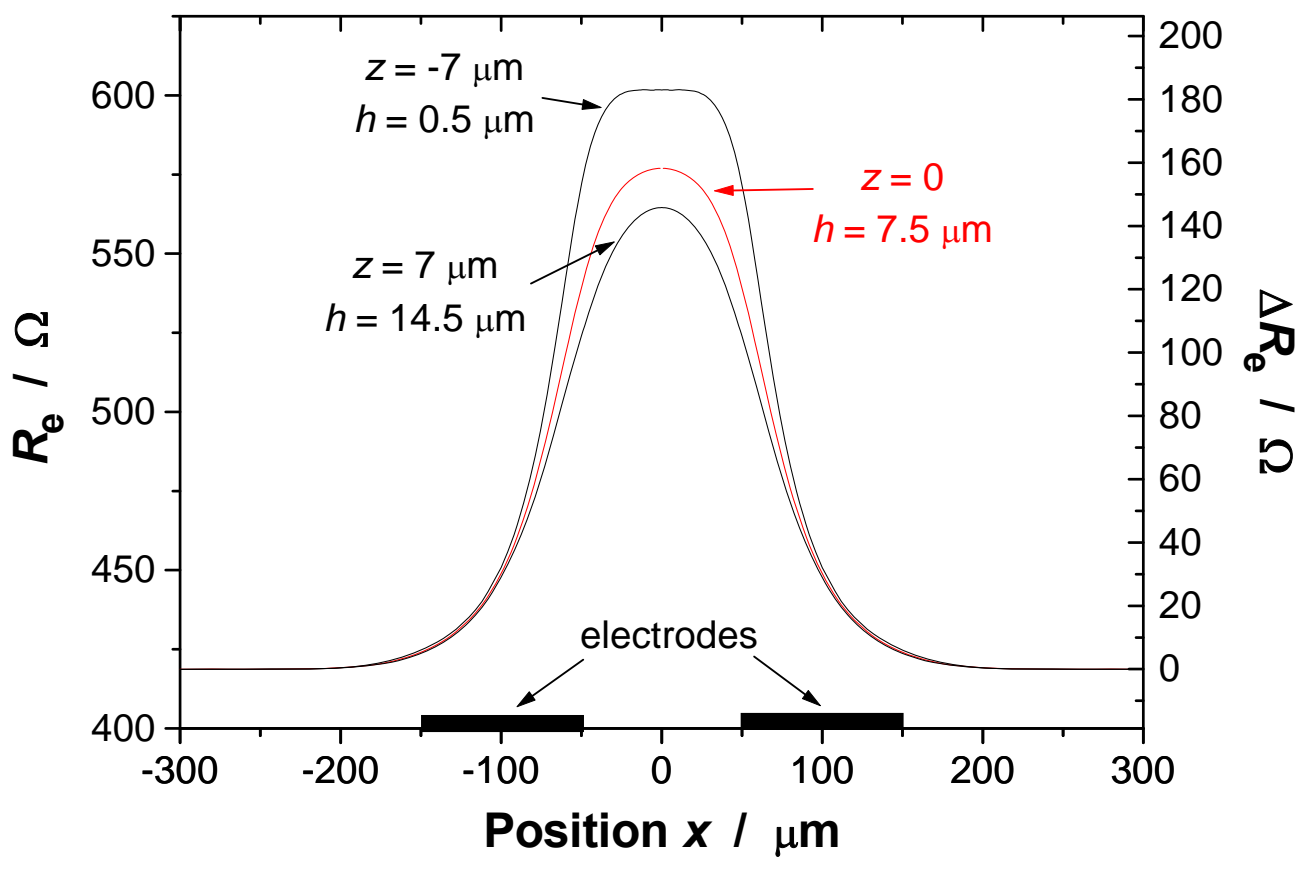

Fig. 4 Variations of $R_{\mathrm{e}}$ and $\Delta R_{\mathrm{e}}$ as a function of the position of the insulating sphere along the flow direction $\mathrm{O} x$ and its vertical position $z\left(y=0, d_{\mathrm{p}}=85 \mu \mathrm{m}\right)$. 


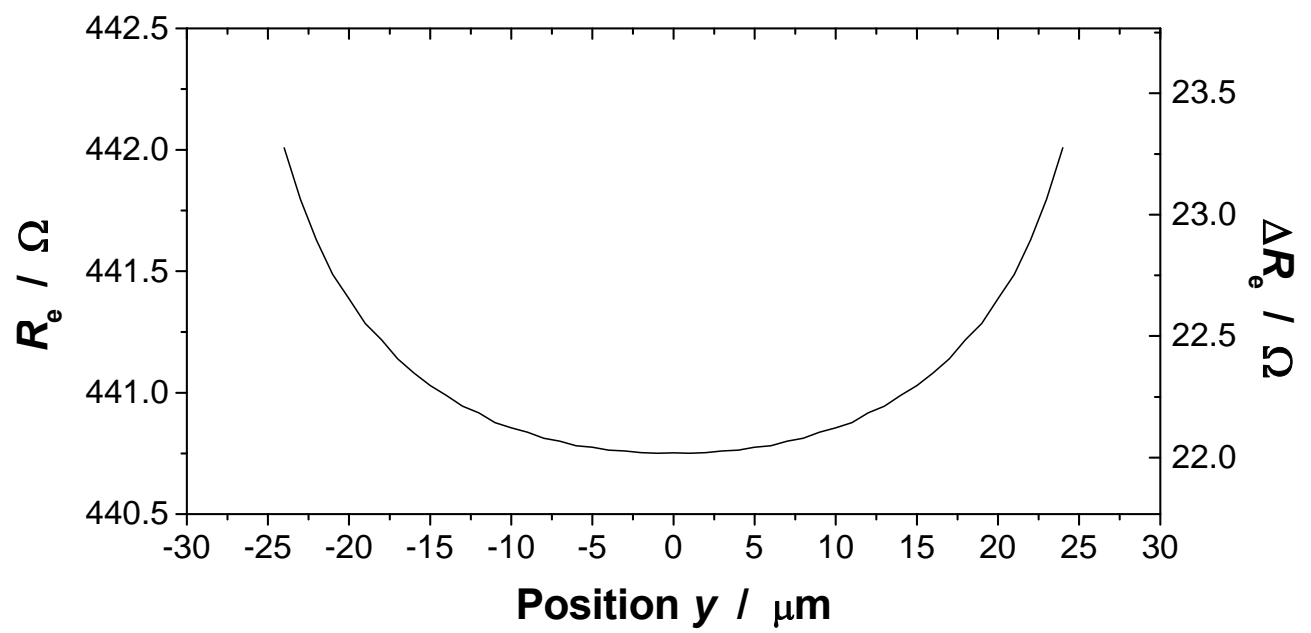

Fig. 5 Variations of $R_{\mathrm{e}}$ and $\Delta R_{\mathrm{e}}$ as a function of the position of the insulating sphere along the $y$ axis $\left(x=0, z=0, d_{\mathrm{p}}=50 \mu \mathrm{m}\right)$. 


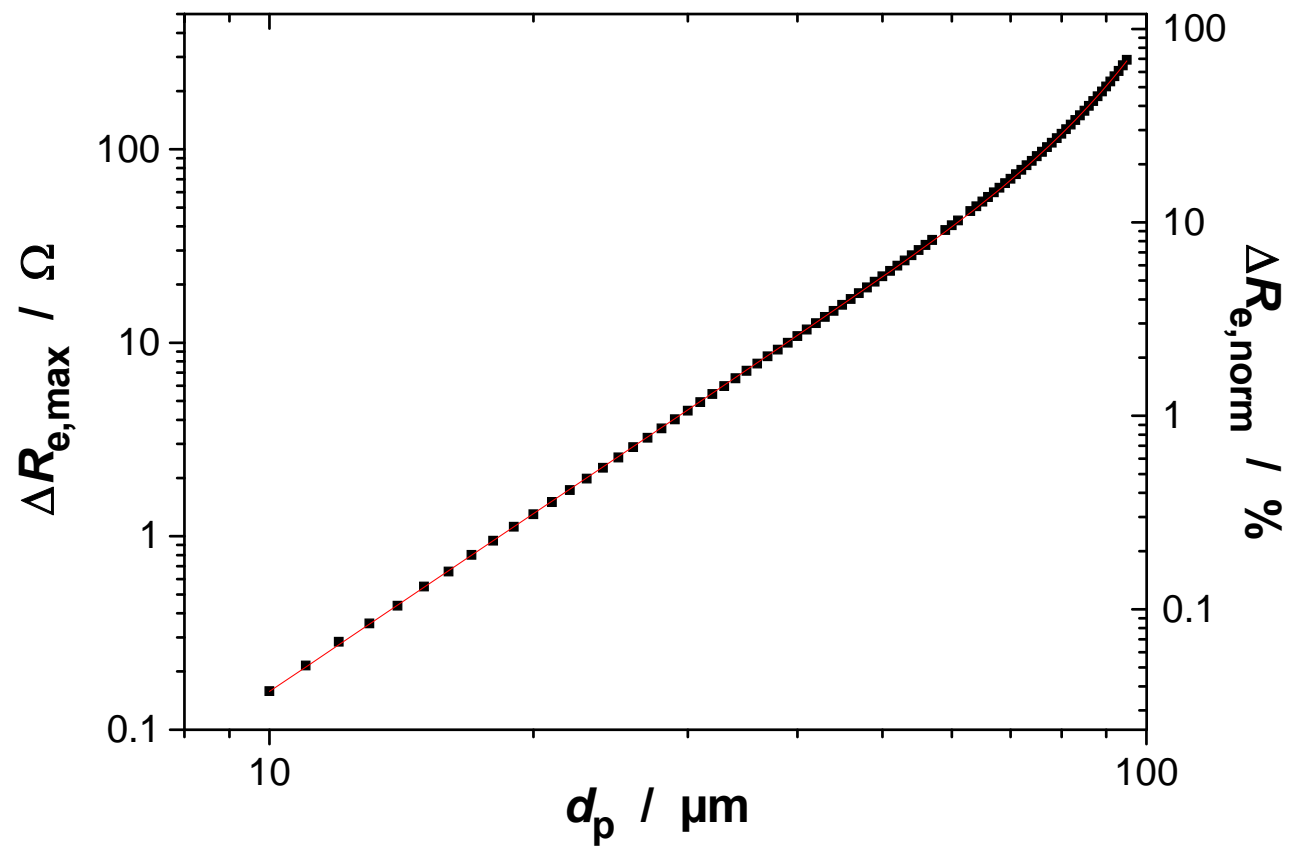

Fig. 6 Variations of $\Delta R_{\mathrm{e}}$ and $\Delta R_{\mathrm{e}, \text { norm }}$ as a function of the diameter of the insulating sphere located at the centre of the channel $(x=y=z=0)$. 


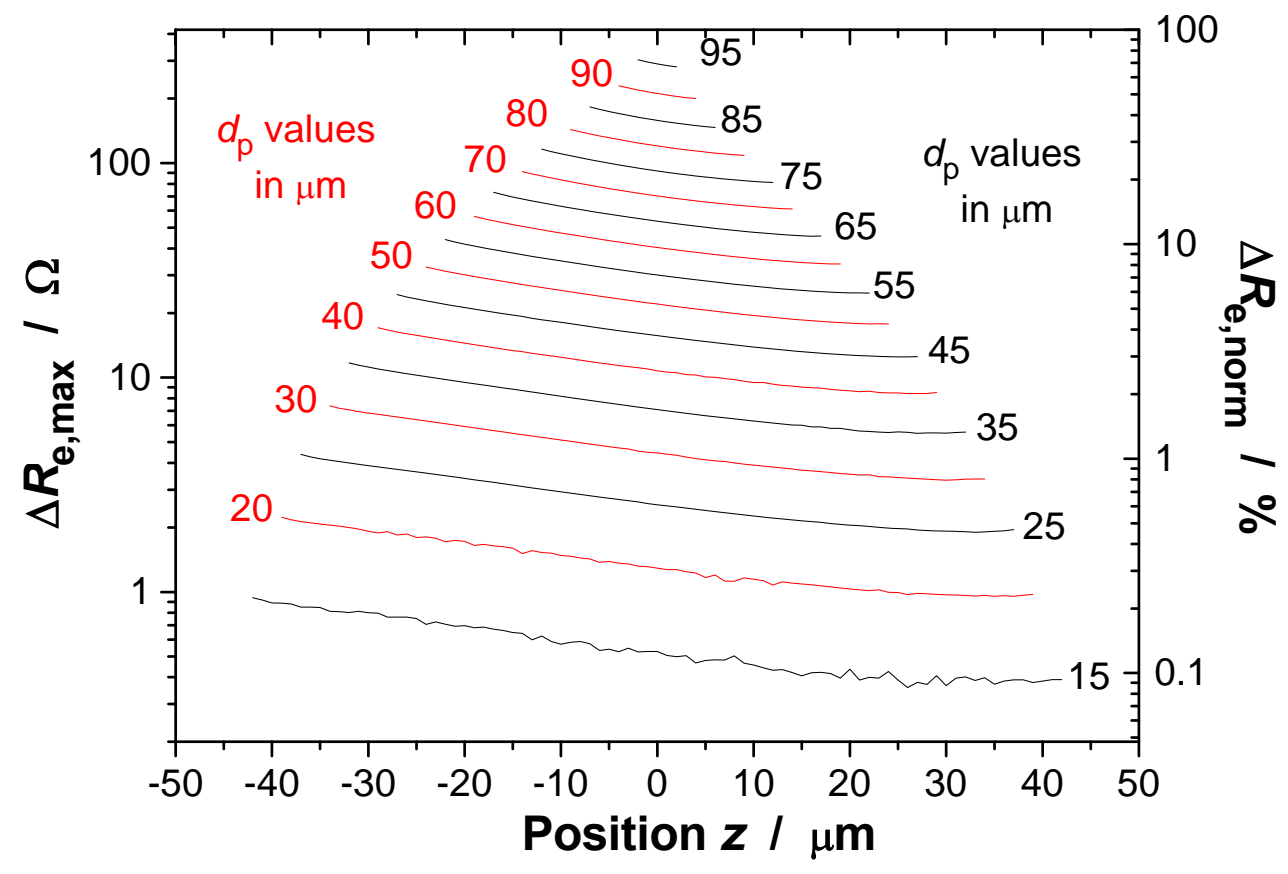

Fig. 7 Variations of $\Delta R_{\mathrm{e}, \max }$ and $\Delta R_{\mathrm{e}, \text { norm }}$ as a function of the vertical position and diameter of the insulating sphere $(x=y=0)$. 


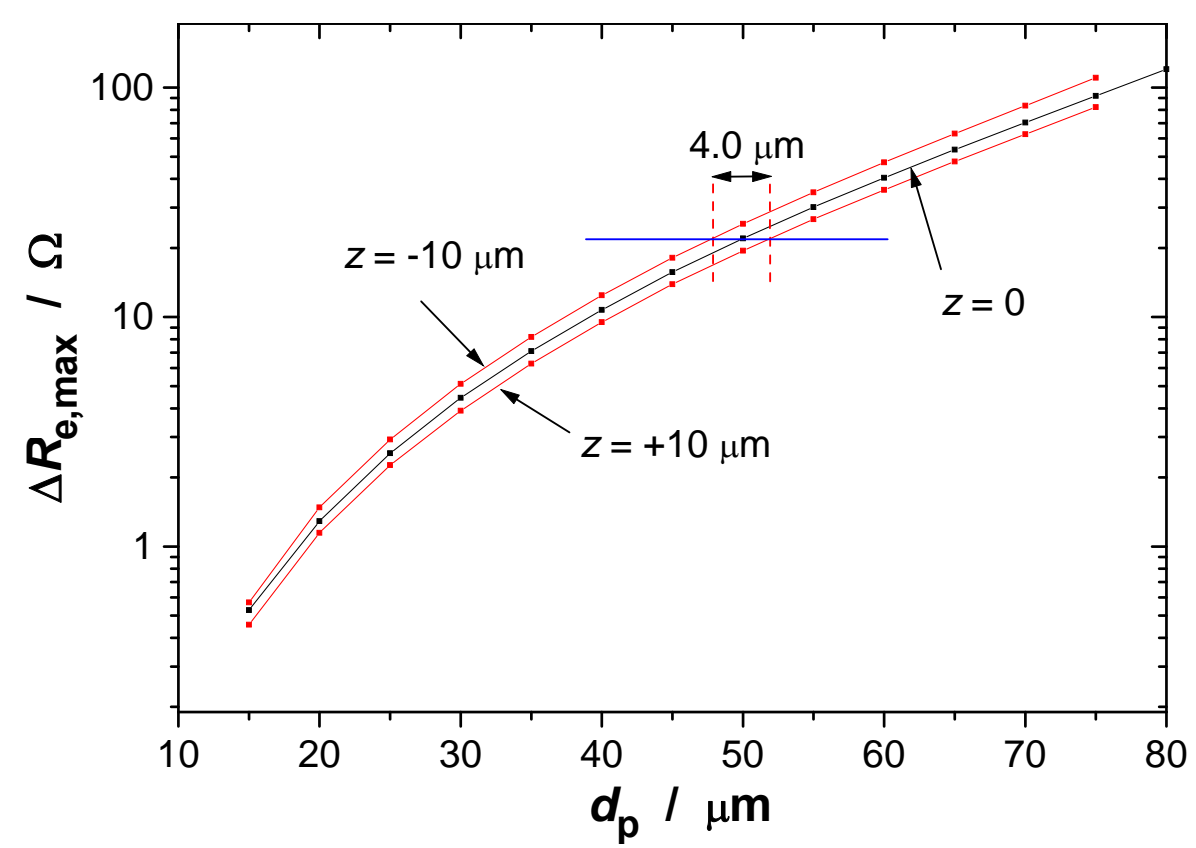

Fig. 8 Estimation of the sphere diameter from the measured value of $\Delta R_{\mathrm{e}, \max }(x=y=0)$. 


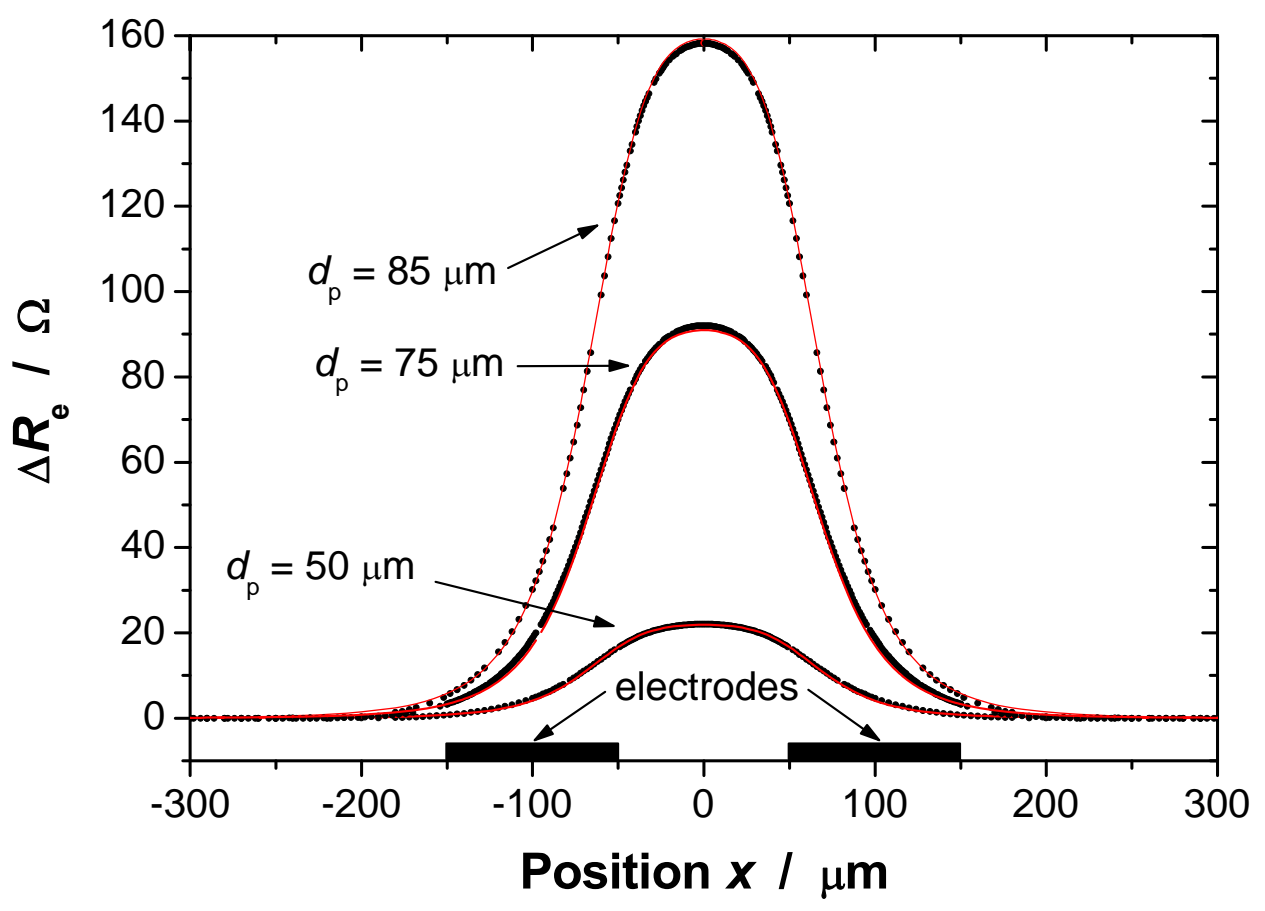

Fig. 9 Values of $\Delta R_{\mathrm{e}}$ as a function of the position of the insulating sphere along the flow direction Ox for $y=z=0$ and different sphere diameters. Solid red lines obey Eq. 8 . 


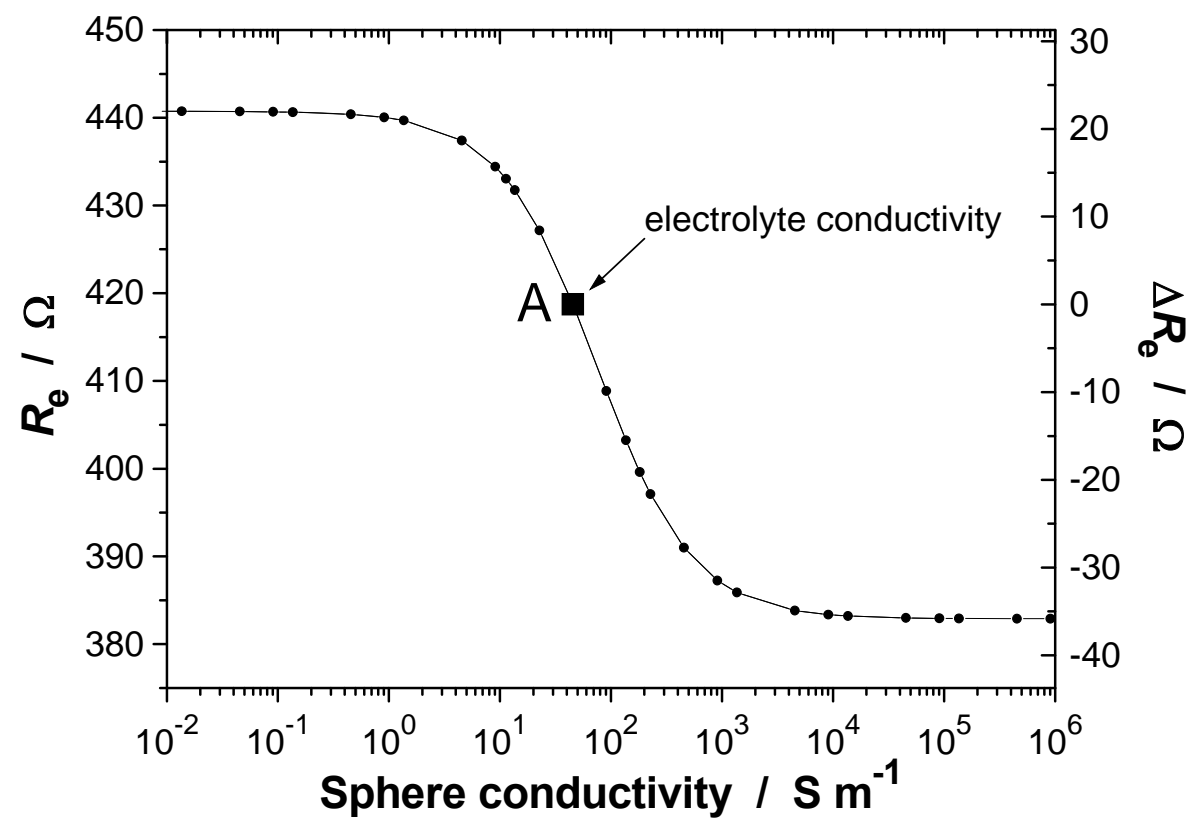

Fig. 10 Variations of $R_{\mathrm{e}}$ and $\Delta R_{\mathrm{e}}$ as a function of the conductivity of a sphere of diameter $50 \mu \mathrm{m}$ located at the centre of the channel $(x=y=z=0)$. 


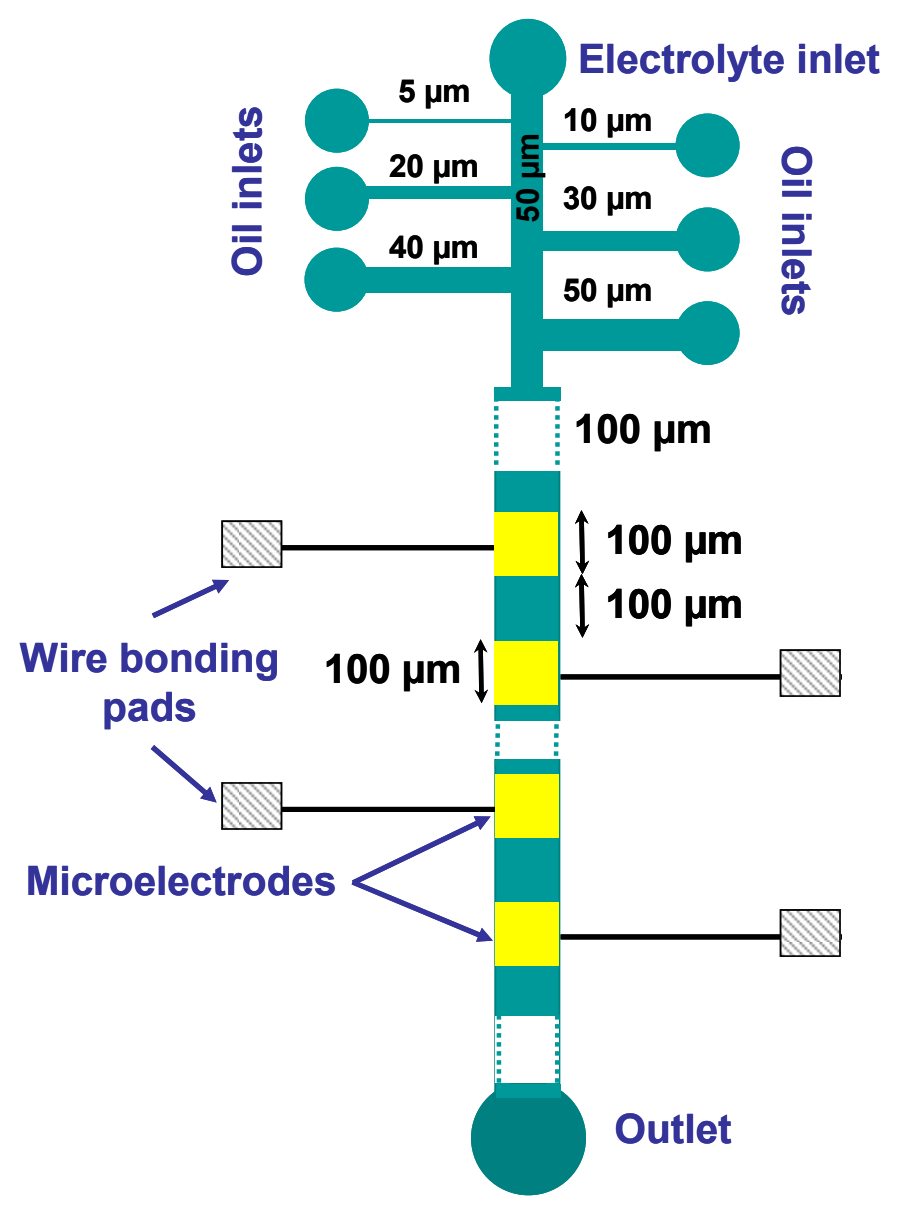

Fig. 11 Schematic illustration of the microfluidic device. 


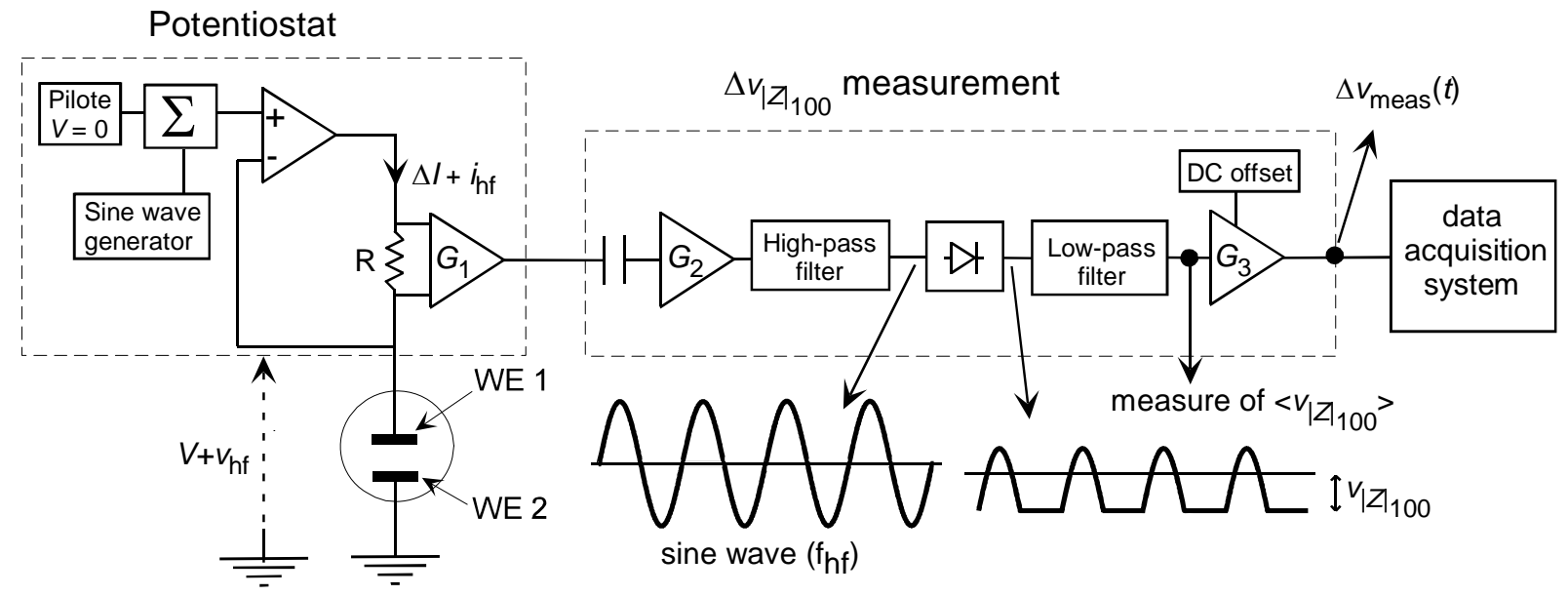

Fig. 12 Experimental setup used for measuring the ER fluctuations. 

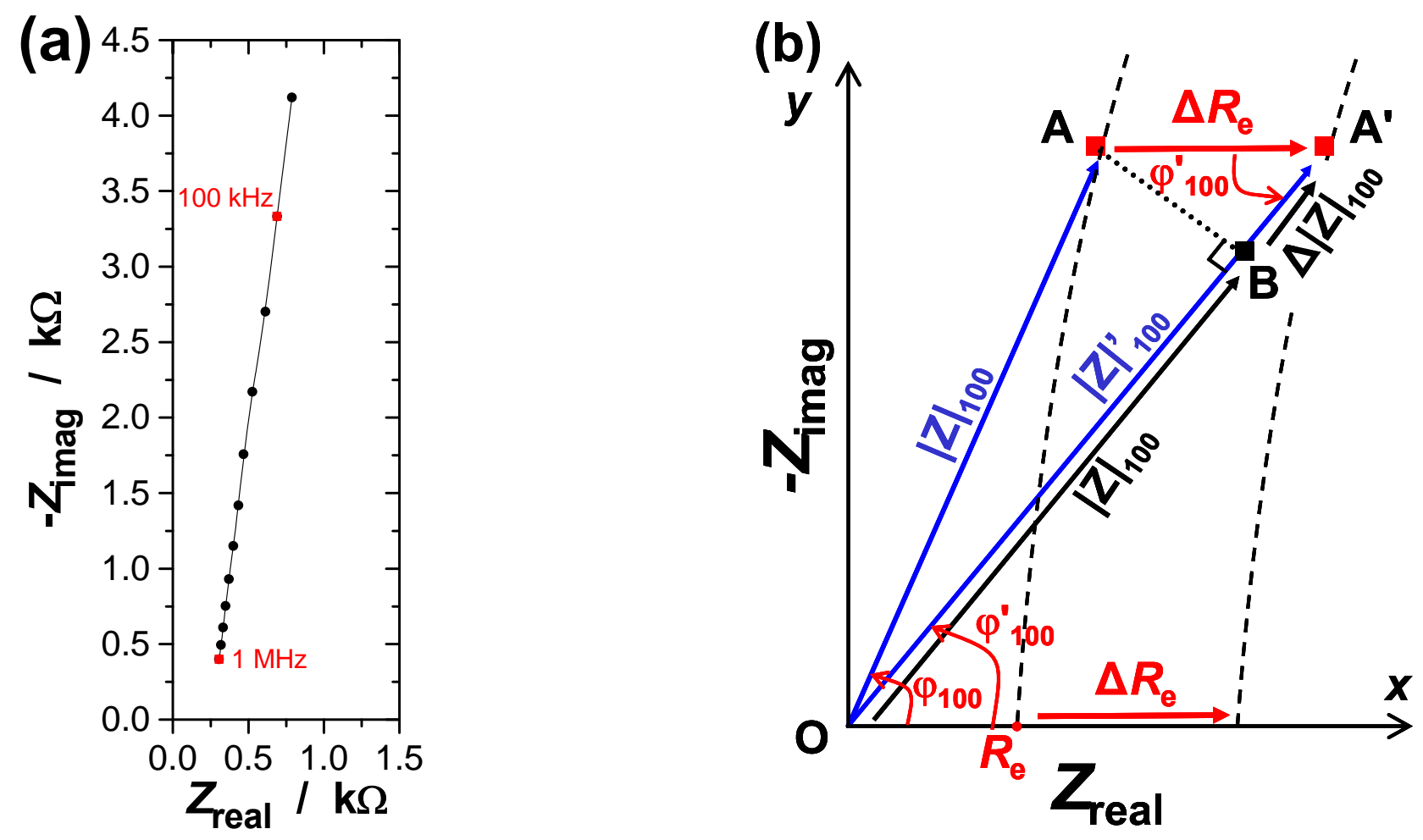

Fig. 13 (a) High-frequency impedance Nyquist diagram of a pair of electrodes $\left(d_{\mathrm{x}}=D=100 \mu \mathrm{m}\right)$ and (b) method for measuring the $\Delta R_{\mathrm{e}}$ fluctuations. 
(a)

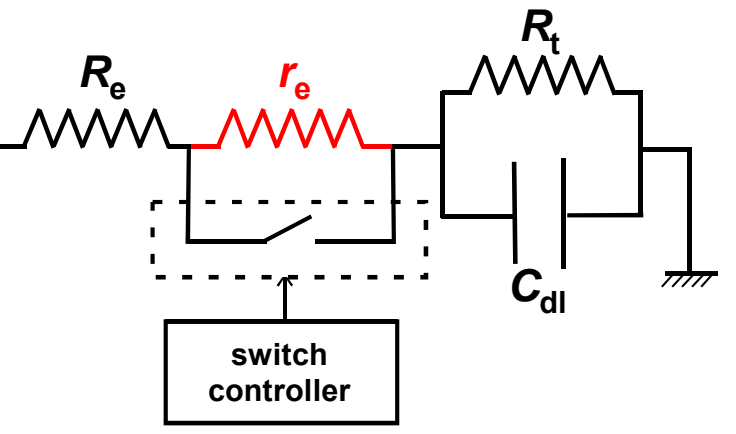

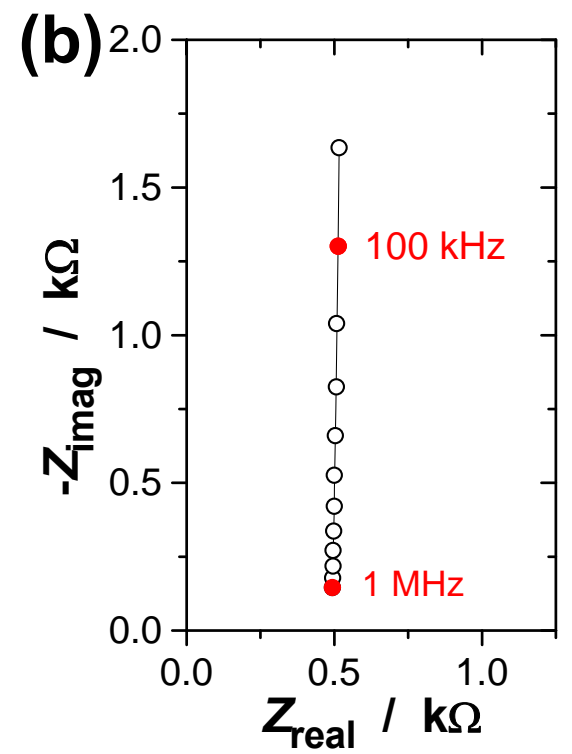

Fig. 14 Dummy cell $\left(R_{\mathrm{e}}=500 \Omega, r_{\mathrm{e}}=200 \Omega, R_{\mathrm{t}}=700 \mathrm{k} \Omega, C_{\mathrm{dl}}=1.2 \mathrm{nF}\right)$ used for for validating the $\Delta R_{\mathrm{e}}$ measurements (a) and corresponding high-frequency impedance Nyquist diagram (b). 

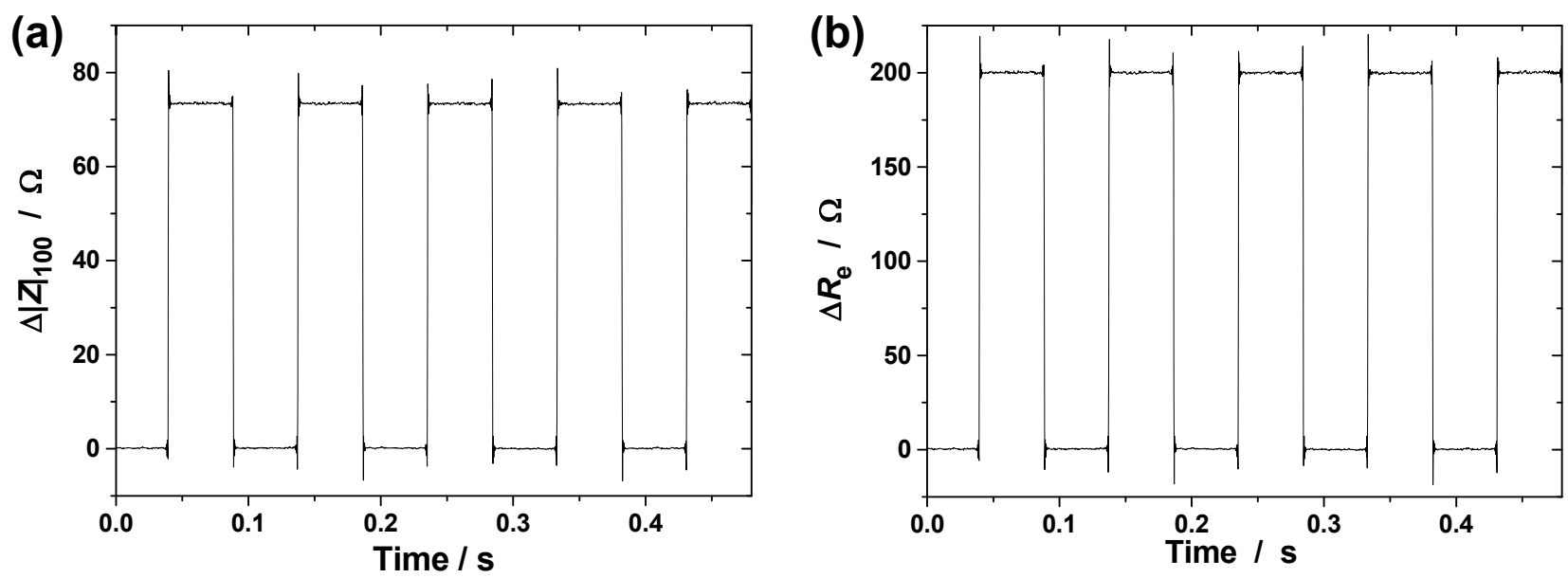

Fig. 15 Variations of $|Z|_{100}$ measured on the dummy cell in Fig. 14a (a) and corresponding variations of $R_{\mathrm{e}}(\mathrm{b})$. 

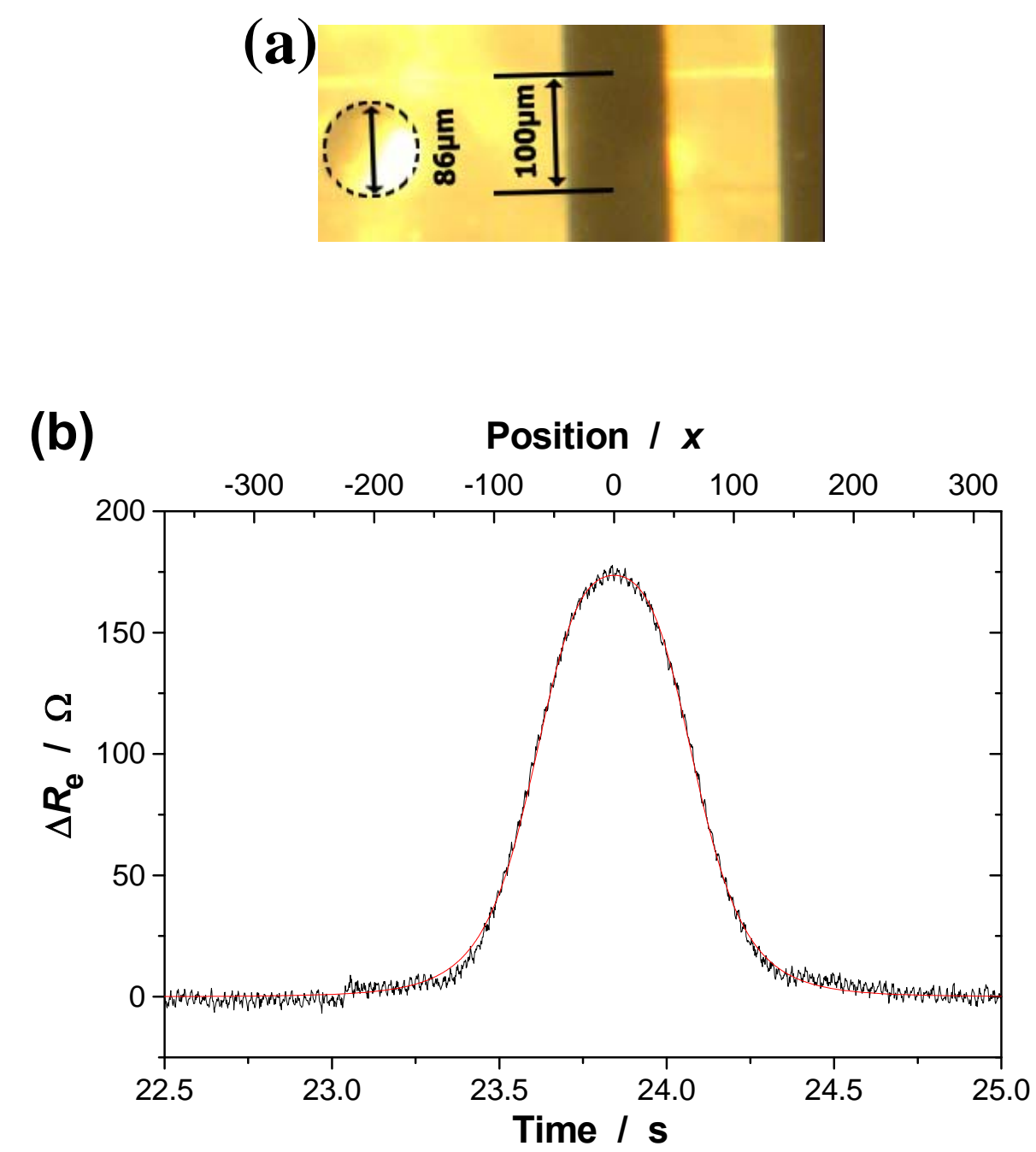

Fig. 16 Image of an oil droplet of $86 \mu \mathrm{m}$ in diameter flowing in the microchannel (a) and corresponding experimental (black) and theoretical (red) $R_{\mathrm{e}}$ variations calculated with Eq. 9 and the fitted parameters $d_{\mathrm{p}}=86.51 \mu \mathrm{m}, t_{0}=23.843 \mathrm{~s}$, and $v=279.1 \mu \mathrm{m} \mathrm{s}^{-1}(\mathrm{~b})$. 


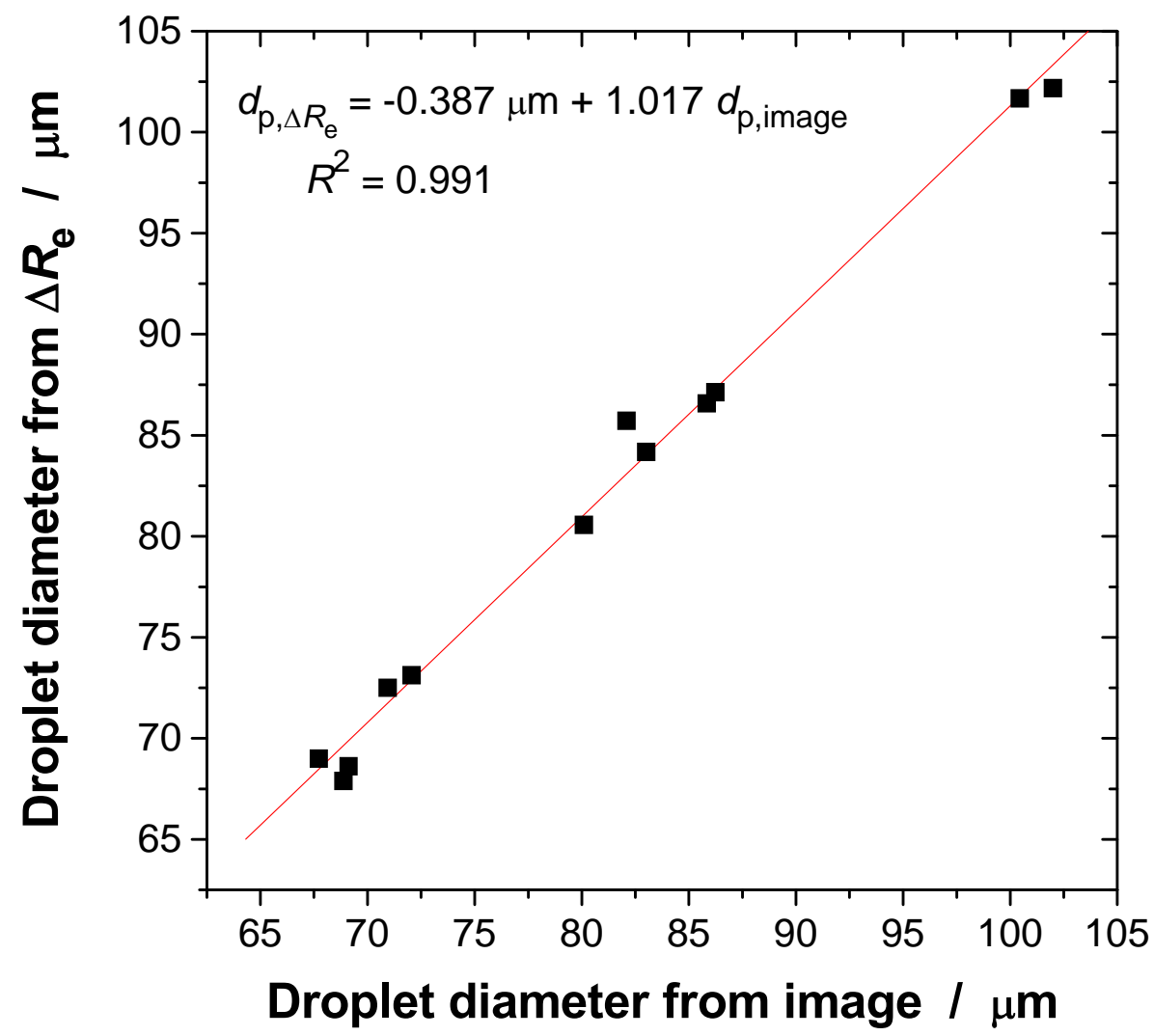

Fig. 17 Droplet diameter estimated from $\Delta R_{\mathrm{e}}$ variations as a function of the droplet diameter estimated from the images. 

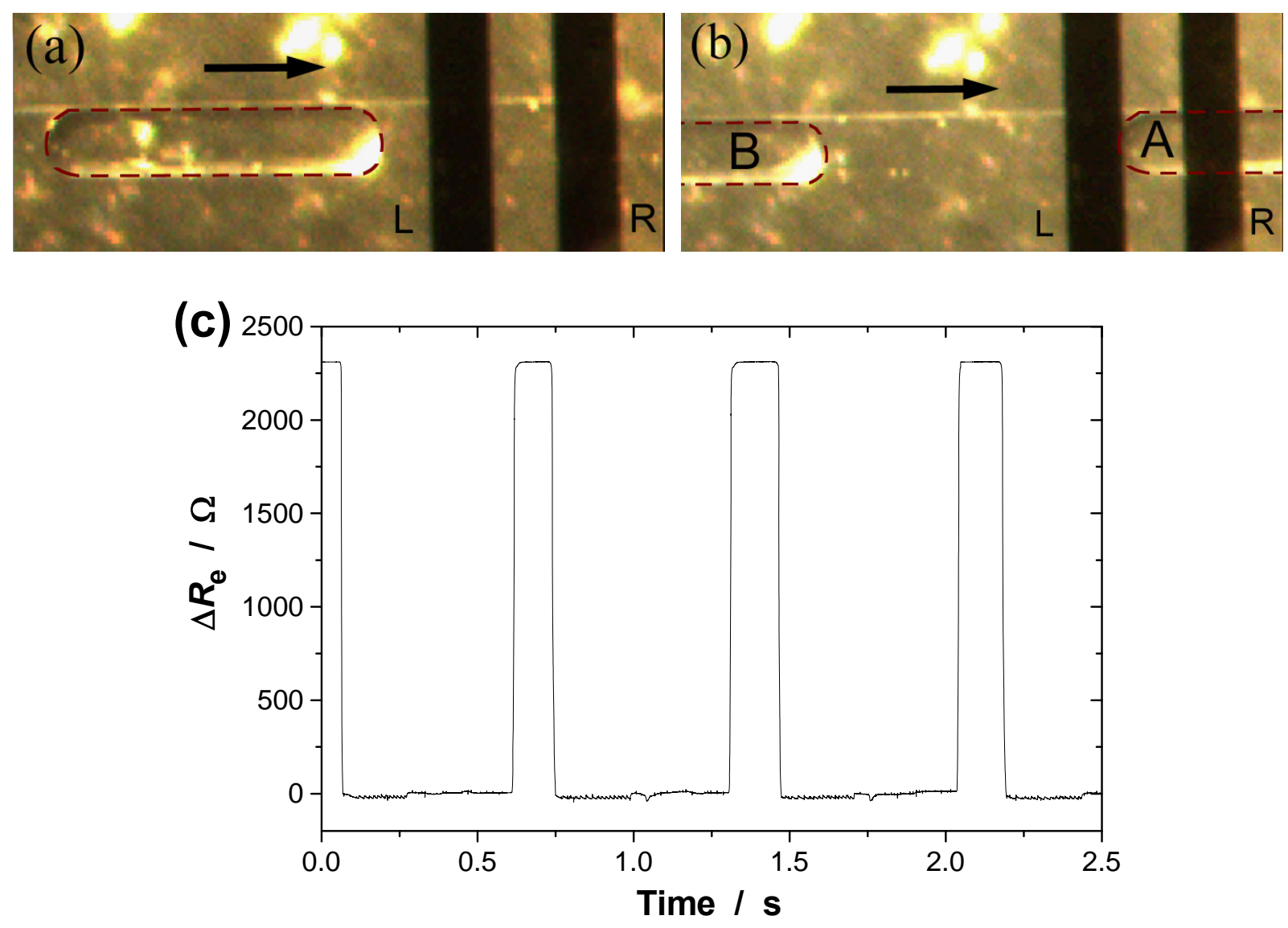

Fig. 18 Images of the passage of oil plugs above the L (left) and R (right) electrodes (a, b) and corresponding $\Delta R_{\mathrm{e}}$ variations (c). 